\title{
Change of universe functors in equivariant stable homotopy theory
}

\author{
by
}

\author{
L. Gaunce Lew is, J r. (Syracuse, N.Y.)
}

\begin{abstract}
One striking difference between nonequivariant and equivariant stable homotopy is that, in the equivariant context, one must specify those representations with respect to which spectra are to be stable. One may specify stability with respect only to trivial representations (thereby obtaining what is often called the naive equivariant stable category), with respect to all representations (thereby obtaining the full equivariant stable category), or with respect to any intermediate collection of representations closed under direct sums. The chosen family of representations is usually described by specifying an indexing universe. Change of universe functors transform spectra stable with respect to one set of representations into spectra stable with respect to a second set of representations. This is done either by restriction (that is, by forgetting the stability with respect to some representations) or by induction (that is, by altering the spectra so that they become stable with respect to a larger class of representations). The impact of these transformations on the equivariant homotopy groups of spectra should be viewed as an equivariant generalization of the passage between unstable and stable homotopy groups in the nonequivariant context. Three results concerning this impact are given. One describes when change of universe functors are isomorphisms of categories. The second completely describes the impact of an arbitrary induction functor on the first nonvanishing homotopy groups of a bounded-below spectrum. The third gives a spectral sequence which describes the behavior of an arbitrary induction functor on all the homotopy groups of an arbitrary spectrum.
\end{abstract}

Introduction. This paper continues the study begun in [13] and [12] of the equivariant Hurewicz and suspension maps. In [12], it was shown that direct equivariant generalizations of the Freudenthal suspension theorem necessarily suffer from at least one of two defects - either their hypotheses are unduly restrictive, or they describe the effect of suspension only on the bottom nonvanishing homotopy groups rather than on the homotopy groups in a range of dimensions. One of the purposes of the present paper is to introduce a spectral sequence, promised in [12], which ameliorates this

1991 Mathematics Subject Classification: Primary 55M35, 55P42, 55P91, 55T99, 57S15; Secondary 55N91, 55P20, 55Q10, 55Q91. 
situation by providing a way of studying the effect of suspension in precisely that range of dimensions where one might expect to be able to understand it based on the nonequivariant Freudenthal suspension theorem.

This spectral sequence is most easily constructed by studying a portion of equivariant homotopy theory that lies between the unstable and stable realms and has no exact nonequivariant analog. To understand the nature of this in-between realm, recall that, modulo a couple of technical points, the nonequivariant stable category may be regarded as the category obtained from the homotopy category $h \mathcal{T}$ of based spaces by making the suspension functor invertible. When we move from the homotopy category of based spaces to the homotopy category $h G \mathcal{T}$ of based $G$-spaces, where $G$ is a compact Lie group, then there is no longer a single suspension functor, but rather there is a "suspension by $W$ " functor $\Sigma^{W}$ associated with each finite-dimensional representation $W$ of $G$. Thus, rather than expecting just a single $G$-stable category, one ought to expect a family of what might be called semi-stable categories. Associated with any collection $\mathcal{U}$ of (isomorphism classes of) finite-dimensional $G$-representations, there should be a category, which might be called the $G$-U- -semi-stable category, that is obtained from $h G \mathcal{T}$ by inverting the suspension functors $\Sigma^{W}$ associated with the representations $W$ in $\mathcal{U}$. Among this family of categories there is an obvious maximal one in which suspension by every finite-dimensional $G$-representation $W$ is invertible. This maximal category is the real $G$-stable category. The other categories form the in-between realm in which we work to construct our spectral sequence.

Due to the way in which $G$-semi-stable categories are constructed [18], they are actually indexed on certain infinite-dimensional $G$-representations, called $G$-universes, rather than on the collections $\mathcal{U}$ of $G$-representations suggested above. A countably infinite-dimensional inner product space $U$ on which $G$ acts by isometries is a $G$-universe if it contains both the trivial representation and infinitely many copies of each of its finite-dimensional subrepresentations. The semi-stable category associated with a $G$-universe $U$ is denoted by $\widetilde{h} G \mathcal{S} U$; in [18], it is referred to as the G-stable category indexed on $U$. The objects of $\widetilde{h} G \mathcal{S} U$ are referred to as $G$-spectra indexed on $U$. For each finite-dimensional $G$-representation $W$, there are adjoint functors $\Sigma^{W}$ and $\Omega^{W}$ on $\widetilde{h} G \mathcal{S} U$ which are, in an appropriate sense, the extensions to $\widetilde{h} G \mathcal{S} U$ of the suspension and loop functors $\Sigma^{W}$ and $\Omega^{W}$ on $h G \mathcal{T}$. The functor $\Sigma^{W}$ is invertible on $\widetilde{h} G \mathcal{S} U$ if $W$ is contained in $U$. Thus, $\widetilde{h} G \mathcal{S} U$ may be thought of as the semi-stable category associated with the collection $\mathcal{U}$ of those finite-dimensional $G$-representations which are subrepresentations of $U$. If $W$ is not a subrepresentation of $U$, then $\Sigma^{W}$ need not be an invertible functor on $\widetilde{h} G \mathcal{S} U$. One of the two primary purposes of this article is to describe the behavior of $\Sigma^{W}$ for such $W$. Our results on the func- 
tors $\Sigma^{W}: \widetilde{h} G \mathcal{S} U \rightarrow \widetilde{h} G \mathcal{S} U$ should be thought of as generalizations of the Freudenthal suspension theorem since they describe the effect of suspension by a representation $W$ with respect to which the objects of $\widetilde{h} G \mathcal{S} U$ have not been stabilized. The spectral sequence described earlier which describes the behavior of the space-level suspension functor $\Sigma^{W}$ is derived from a spectral sequence which describes the behavior of the suspension functor $\Sigma^{W}$ on an appropriately chosen semi-stable category $\widetilde{h} G \mathcal{S} U$.

Associated with each linear $G$-isometry $\iota: U \rightarrow U^{\prime}$, there is a pair $\iota_{*}: \widetilde{h} G \mathcal{S} U \rightarrow \widetilde{h} G \mathcal{S} U^{\prime}, \iota^{*}: \widetilde{h} G \mathcal{S} U^{\prime} \rightarrow \widetilde{h} G \mathcal{S} U$ of adjoint functors which are called change of universe functors [18]. The right adjoint $\iota^{*}$ "forgets" that the objects of $\widetilde{h} G \mathcal{S} U^{\prime}$ are stable with respect to those $G$-representations $W$ that are contained in $U^{\prime}$ but not in $U$. The left adjoint $\iota_{*}$ stabilizes the objects of $\widetilde{h} G \mathcal{S} U$ with respect to precisely the same representations $W$. The second primary purpose of this paper is to describe the effects of $\iota_{*}$. The functor $\iota_{*}$ can be thought of, in a sense that we make precise in Section 5, as a kind of colimit of the suspension functors $\Sigma^{W}$ for those $W$ which are contained in $U^{\prime}$ but not in $U$. Thus, our results describing the behavior of $\iota_{*}$ should be thought of as equivariant generalizations of the Freudenthal suspension theorem.

In a sense, each suspension functor $\Sigma^{W}: \widetilde{h} G \mathcal{S} U \rightarrow \widetilde{h} G \mathcal{S} U$ and each change of universe functor $\iota_{*}: \widetilde{h} G \mathcal{S} U \rightarrow \widetilde{h} G \mathcal{S} U^{\prime}$ is one small piece of the long road between the category of $G$-spaces and the $G$-stable category. From this point of view, the results presented here should be of interest for the insights they provide on the gap between the unstable and stable realms in equivariant homotopy theory. A second, more subtle, reason for interest in these results is that they ought to provide some insight into the unsolved problem of constructing useful geometric models for the equivariant loop spaces $\Omega^{W} \Sigma^{W} X$ and infinite loop space $\Omega^{\infty} \Sigma^{\infty} X$ associated with a $G$-space $X$ in the context where $G$ is a compact Lie, but not finite, group. Our results may be thought of as providing an almost entirely algebraic description of the homotopy groups of the spaces $\Omega^{W} \Sigma^{W} X$ and $\Omega^{\infty} \Sigma^{\infty} X$ in terms of the homotopy groups of the $G$-space $X$. These descriptions are essentially independent of whether or not $G$ is finite. When $G$ is finite, useful geometric models of the spaces $\Omega^{W} \Sigma^{W} X$ and $\Omega^{\infty} \Sigma^{\infty} X$ already exist [3, 8]. Our hope is that, by studying the relation between our algebraic results and the known geometric models in the finite case, we may gain enough insight about the connections between the algebra and the geometry to work from our algebraic results in the nonfinite case to the necessary structure of a geometric model for that case.

The first section of this paper begins with a review of some of the basic terminology of equivariant stable homotopy theory and concludes with the statements of our main results. The second section discusses the implica- 
tions of a suspension spectral sequence introduced in the first section for the space-level equivariant suspension theorem. Most of our results follow from certain properties of equivariant Eilenberg-MacLane spectra. These spectra, and a special type of equivariant $C W$ spectra needed for their construction, are discussed in Section 3. This section also contains the proofs of those of our results which follow trivially from the properties of Eilenberg-MacLane spectra. Our spectral sequences are derived from Postnikov decompositions of equivariant spectra; these are described in Section 4. Section 5 contains a description, which has proved to be computationally useful, of the functors $\iota_{*}$ in terms of suspension functors. Section 6 contains the proof of the one result from Section 1 which does not follow from the properties of Eilenberg-MacLane spectra. Much of our notation, and many basic facts about equivariant stable homotopy theory, are assumed from the first two chapters of [18].

I am indebted to Peter May for questioning me about the implications of the results in [13] and a preliminary version of [12] for equivariant EilenbergMacLane spaces and spectra. It was in pursuing answers to his questions that I discovered most of the results in this paper. I am also indebted to Stefan Waner for providing me a copy of his unpublished work on $G-C W(V)$ complexes [27]. My discussion of $G-C W(\alpha)$ spectra in Section 3 draws heavily on Waner's earlier work.

1. Properties of suspension and change of universe functors in equivariant stable categories. After reviewing some basic concepts and some notation from equivariant homotopy theory, we here summarize our main results on suspension functors $\Sigma^{W}: \widetilde{h} G \mathcal{S} U \rightarrow \widetilde{h} G \mathcal{S} U$ and change of universe functors $\iota_{*}: \widetilde{h} G \mathcal{S} U \rightarrow \widetilde{h} G \mathcal{S} U^{\prime}$. Each of our three main results contains two parts - one applicable to the functors $\Sigma^{W}$ and the other a parallel observation about the functors $\iota_{*}$. The first main result describes when the functors $\Sigma^{W}$ and $\iota_{*}$ are invertible. The second describes the behavior of both types of functors on the lowest-dimensional nonvanishing homotopy groups of bounded-below spectra. The third provides a spectral sequence for each type of functor which completely describes the effect of that functor on equivariant homotopy groups. This section concludes with some observations about the behavior of our spectral sequences in some very simple special cases.

Throughout this paper, groups are assumed to be compact Lie groups, and subgroups are understood to be closed. The notation $K \leq G$ indicates that $K$ is a subgroup of $G$. All topological spaces are assumed to be compactly generated, weak Hausdorff spaces [16, 23, 29]. All $G$-spaces are left $G$-spaces. Whenever possible, the prefix $G$ is omitted from our notations, so 
that by spaces, subspaces, spectra, maps, etc., we mean $G$-spaces, sub- $G$ spaces, $G$-spectra, $G$-maps, etc. If $K$ is a subgroup of $G$ and $Y$ is a $G$-space, then $Y^{K}$ is the $K$-fixed subspace of $Y$. A based $G$-space is a $G$-space $Y$ together with a specified basepoint, which is required to be in $Y^{G}$. If $X$ is an unbased $G$-space, then $X_{+}$denotes the disjoint union of $X$ and a $G$-trivial basepoint.

All $G$-representations are assumed to have a $G$-invariant inner product. If $W$ is a $G$-representation, then $D W, S W$, and $S^{W}$ denote its unit disk, its unit sphere, and its one-point compactification, respectively. The basepoint of $S^{W}$ is the point at infinity. The dimension of $W$ over the real numbers is denoted by $|W|$. The trivial $G$-representation of dimension $n$, where $n$ is a nonnegative integer, is denoted by $n$. In particular, the zero-dimensional trivial representation is denoted by 0 . If $V$ and $W$ are two $G$-representations, then $V+W$ denotes their direct sum.

Recall that any $G$-universe $U$ is assumed to contain a trivial representation. This ensures that suspension by the trivial representation is invertible in $\widetilde{h} G \mathcal{S} U$ and therefore that $\widetilde{h} G \mathcal{S} U$ shares some of the most basic properties of the nonequivariant stable category. In particular, if $Y$ and $Z$ are in $\widetilde{h} G \mathcal{S} U$, then the set $[Y, Z]_{G}^{U}$ of morphisms from $Y$ to $Z$ in $\widetilde{h} G \mathcal{S} U$ is an abelian group. Moreover, cofibre sequences in $\widetilde{h} G \mathcal{S} U$ are, up to signs, also fibre sequences (see Section III.2 of [18]) and therefore give rise to long exact sequences in both homology and cohomology. Let $\widetilde{h} G \mathcal{T}$ be the category obtained from $h G \mathcal{T}$ by inverting weak equivalences. Then, for any $G$-universe $U$, there is a pair of functors $\Sigma^{\infty}: \widetilde{h} G \mathcal{T} \rightarrow \widetilde{h} G \mathcal{S} U$ and $\Omega^{\infty}: \widetilde{h} G \mathcal{S} U \rightarrow \widetilde{h} G \mathcal{T}$, with $\Sigma^{\infty}$ left adjoint to $\Omega^{\infty}$, which assign to each $G$-space its suspension spectrum and to each $G$-spectrum indexed on $U$ its associated infinite loop space. We denote these functors by $\Sigma_{U}^{\infty}$ and $\Omega_{U}^{\infty}$ when it is necessary to specify the universe $U$.

We have already noted that, if $U$ is a $G$-universe and $W$ is a finitedimensional $G$-representation contained in $U$, then the functor $\Sigma^{W}$ is invertible on $\widetilde{h} G \mathcal{S} U$. However, this functor may be invertible even when $W$ is not contained in $U$. Our first main result describes the precise conditions under which the functors $\Sigma^{W}$ and $\iota_{*}$ are invertible. This description is in terms of a preorder, and its associated equivalence relation, on the category of $G$-representations.

Definition 1.1. Let $V$ and $W$ be $G$-representations. Then $V$ is contained in $W$ up to $G$-orbits if, for each pair of subgroups $K \leq H$ in $G$, the orbit $H / K H$-imbeds in $W$ whenever it $H$-imbeds in $V$. The representations $V$ and $W$ are $G$-orbit equivalent if, for each pair of subgroups $K \leq H$ in $G$, the orbit $H / K H$-imbeds in $V$ if and only if it $H$-imbeds in $W$-that is, each of $V$ and $W$ is contained in the other up to $G$-orbits. 
TheOREM 1.2. (a) Let $U$ be a $G$-universe and $W$ be a finite-dimensional $G$-representation. Then the functor $\Sigma^{W}$ is an equivalence of categories on $\widetilde{h} G \mathcal{S} U$ if and only if $W$ is contained in $U$ up to $G$-orbits.

(b) Let $\iota: U \rightarrow U^{\prime}$ be a linear $G$-isometry between $G$-universes. Then the functor $\iota_{*}: \widetilde{h} G \mathcal{S} U \rightarrow \widetilde{h} G \mathcal{S} U^{\prime}$ is an equivalence of categories if and only if $U$ and $U^{\prime}$ are $G$-orbit equivalent.

Remarks 1.3. (a) Note that the "if" portion of part (a) of the theorem follows from part (b). Let $U^{\prime}$ be the direct sum of the $G$-universe $U$ and countably infinitely many copies of the finite-dimensional $G$-representation $W$, and let $\iota: U \rightarrow U^{\prime}$ be the obvious inclusion of $U$ into $U^{\prime}$. If $W$ is contained in $U$ up to $G$-orbits, then $U$ and $U^{\prime}$ are $G$-orbit equivalent and $\iota_{*}$ is an equivalence of categories. The natural isomorphism $\iota_{*} \Sigma^{W} \cong \Sigma^{W} \iota_{*}$ (see Proposition II.1.4 of [18]) then implies that $\Sigma^{W}$ must be invertible on $\widetilde{h} G \mathcal{S} U$ because it is invertible on $\widetilde{h} G \mathcal{S} U^{\prime}$. The remainder of this theorem is proved in Section 6.

(b) Whenever $\Sigma^{W}$ and $\iota_{*}$ are equivalences of categories, their right adjoints $\Omega^{W}$ and $\iota^{*}$ are, by a standard formal argument, their inverse equivalences. However, to emphasize the invertibility of $\Sigma^{W}$, we denote the inverse equivalence to $\Sigma^{W}$ by $\Sigma^{-W}$ rather than $\Omega^{W}$.

(c) Even if the universes $U$ and $U^{\prime}$ are $G$-orbit equivalent, there need not be an equivariant linear isometry from $U$ to $U^{\prime}$ (or from $U^{\prime}$ to $U$ ). However, if the two are $G$-orbit equivalent, then both are $G$-orbit equivalent to $U \oplus U^{\prime}$ and both imbed, via equivariant linear isometries, into $U \oplus U^{\prime}$. It follows that the $G$-stable categories $\widetilde{h} G \mathcal{S} U$ and $\widetilde{h} G \mathcal{S} U^{\prime}$ are equivalent, since both are equivalent to $\widetilde{h} G \mathcal{S}\left(U \oplus U^{\prime}\right)$.

(d) The obvious nontrivial example of universes that are $G$-orbit equivalent occurs when $G$ is $\mathbb{Z} / p$, for some odd prime $p$ greater than 3 . Let $W$ and $W^{\prime}$ be two nontrivial irreducible $G$-representations that are not equivalent. Let $U$ be the direct sum of infinitely many copies of both $W$ and the trivial representation, and $U^{\prime}$ be the direct sum of infinitely many copies of both $W^{\prime}$ and the trivial representation. Clearly, $U$ and $U^{\prime}$ are nonisomorphic $G$-universes which are $G$-orbit equivalent. Further examples of nonisomorphic $G$-orbit equivalent $G$-universes may be constructed using the results of $[26]$ on $G$-representations that have the same dimension function.

In the cases where $\Sigma^{W}$ and $\iota_{*}$ are not equivalences of categories, we would like measure how far they are from equivalences. We do this by studying the effects of these two functors on equivariant stable homotopy groups. If we were working with a complete $G$-universe, then our equivariant homotopy groups would be graded on the full real representation $\operatorname{ring} R O(G)$ of $G$. However, since we are working with incomplete universes, we must grade our homotopy groups on an additive submonoid of $R O(G)$. 
DeFINITION 1.4. (a) Recall that the real representation ring $R O(G)$ of $G$ is the Grothendieck group of isomorphism classes of finite-dimensional real $G$-representations. The elements of $R O(G)$ are equivalence classes of formal differences $V-W$ of finite-dimensional $G$-representations $V$ and $W$. If $U$ is a $G$-universe, then $R^{+}(G, U)$ is the additive submonoid of $R O(G)$ consisting of those elements which can be represented by a formal difference of the form $V-W$ such that $V$ and $W$ are finite-dimensional $G$-representations and $W$ is contained in $U$ up to $G$-orbits. If $U$ is a trivial $G$-universe, then $R O^{+}(G, U)$ is the submonoid of $R O(G)$ generated by the formal differences $V-n$, for $n \in \mathbb{Z}$. If $U$ is a complete $G$-universe, then $R O^{+}(G, U)$ is $R O(G)$. Observe that, for any universe $U, R O^{+}(G, U)$ contains the submonoid $\mathbb{Z}$ of trivial virtual representations.

(b) If $\alpha$ is an element of $R O^{+}(G, U)$ represented by the formal difference $V-W$, where $W$ is contained up to $G$-orbits in $U$, then the sphere $S^{\alpha}$ (or $S_{U}^{\alpha}$ if we need to specify the universe) of dimension $\alpha$ in $\widetilde{h} G \mathcal{S} U$ is defined to be the spectrum $\Sigma^{-W} \Sigma^{\infty} S^{V}$. This sphere is uniquely defined up to isomorphism in $\widetilde{h} G \mathcal{S} U$. If there is a linear isometry $\iota: U \rightarrow U^{\prime}$ between the universes $U$ and $U^{\prime}$, then $R O^{+}(G, U)$ is a submonoid of $R O^{+}\left(G, U^{\prime}\right)$. Thus, if $\alpha$ is in $R O^{+}(G, U)$, then we have spheres $S_{U}^{\alpha}$ and $S_{U^{\prime}}^{\alpha}$ in $\widetilde{h} G \mathcal{S} U$ and $\widetilde{h} G \mathcal{S} U^{\prime}$, respectively. There are natural isomorphisms $\iota_{*} \Sigma_{U}^{\infty} \cong \Sigma_{U^{\prime}}^{\infty}$ and $\iota_{*} \Sigma^{V} \cong \Sigma^{V} \iota_{*}$ (see Proposition II.1.4 of [18]), which together provide an isomorphism $\iota_{*} S_{U}^{\alpha} \cong S_{U^{\prime}}^{\alpha}$.

(c) If $Z$ is a $G$-spectrum indexed on the universe $U, K \leq G$, and $\alpha \in R O^{+}(G, U)$, then the $\alpha$ th stable homotopy group $\pi_{\alpha}^{K, U} Z$ of $Z$ with respect to $K$ is the abelian group $\left[S_{U}^{\alpha}, Z\right]_{K}^{U}$. This group is isomorphic to $\left[S_{U}^{\alpha} \wedge G / K_{+}, Z\right]_{G}^{U}$ by Theorem II.4.7 and Lemma II.4.8 of [18]. If $W$ is a $G$-representation, then the suspension functor $\Sigma^{W}$ induces a natural homomorphism

$$
\sigma_{W}^{K}: \pi_{\alpha}^{K, U} Z \rightarrow \pi_{\alpha+W}^{K, U} \Sigma^{W} Z
$$

Similarly, if $\iota: U \rightarrow U^{\prime}$ is a linear isometry, the functor $\iota_{*}$ and the isomorphism $\iota_{*} S_{U}^{\alpha} \cong S_{U^{\prime}}^{\alpha}$ induce a natural homomorphism

$$
\sigma_{\iota}^{K}: \pi_{\alpha}^{K, U} Z \rightarrow \pi_{\alpha}^{K, U^{\prime}} \iota_{*} Z .
$$

Roughly speaking, our measure of the extent to which the functors $\Sigma^{W}$ and $\iota_{*}$ fail to be equivalences of categories is the extent to which the homomorphisms $\sigma_{W}^{K}$ and $\sigma_{\iota}^{K}$ defined above fail to be isomorphisms. The main thrust of the work in [12] on the space-level equivariant suspension map is that, in order to describe the behavior of this map, it is necessary to introduce a generalization of the notion of a Mackey functor which captures the full algebraic structure carried by the equivariant homotopy groups of a $G$-space. The same approach provides the insight needed to understand 
the behavior of the maps $\sigma_{W}^{K}$ and $\sigma_{\iota}^{K}$. Thus, we now introduce the notion of a $U$ - $\alpha$-Mackey functor and show that the collection of homotopy groups $\pi_{\alpha}^{K, U} Z$, for $K \leq G$, forms such an object.

Definition 1.5. (a) If $\alpha \in R O^{+}(G, U)$, then the $U$ - $\alpha$-Burnside category $\mathfrak{B}_{G}^{U}(\alpha)$ has, as its objects, the orbits $G / K$, for $K \leq G$. The collection of morphisms in $\mathfrak{B}_{G}^{U}(\alpha)$ from the orbit $G / K$ to the orbit $G / J$ is the abelian group $\left[S_{U}^{\alpha} \wedge G / K_{+}, S_{U}^{\alpha} \wedge G / J_{+}\right]_{G}^{U}$. Composition of morphisms is defined as in $\widetilde{h} G \mathcal{S} U$. Since composition is linear in either variable, $\mathfrak{B}_{G}^{U}(\alpha)$ is an Ab-category.

(b) If $\alpha \in R O^{+}(G, U)$, then a $U$ - $\alpha$-Mackey functor is a contravariant additive functor from $\mathfrak{B}_{G}^{U}(\alpha)$ to the category $A b$ of abelian groups. The category of $U$ - $\alpha$-Mackey functors, and natural transformations between these functors, is denoted by $\mathcal{M}_{G}^{U}(\alpha)$.

(c) If $W$ is a finite-dimensional $G$-representation, then there is a functor

$$
s_{W}: \mathfrak{B}_{G}^{U}(\alpha) \rightarrow \mathfrak{B}_{G}^{U}(\alpha+W)
$$

which is the identity on objects and which is given on morphisms by the suspension map

$$
\begin{aligned}
{\left[S_{U}^{\alpha} \wedge G / K_{+}, S_{U}^{\alpha} \wedge G / J_{+}\right]_{G}^{U} } & \rightarrow\left[\Sigma^{W} S_{U}^{\alpha} \wedge G / K_{+}, \Sigma^{W} S_{U}^{\alpha} \wedge G / J_{+}\right]_{G}^{U} \\
& \cong\left[S_{U}^{\alpha+W} \wedge G / K_{+}, S_{U}^{\alpha+W} \wedge G / J_{+}\right]_{G}^{U} .
\end{aligned}
$$

Precomposition with $s_{W}$ produces a functor $s_{W}^{*}: \mathcal{M}_{G}^{U}(\alpha+W) \rightarrow \mathcal{M}_{G}^{U}(\alpha)$. It has a left adjoint $s_{*}^{W}: \mathcal{M}_{G}^{U}(\alpha) \rightarrow \mathcal{M}_{G}^{U}(\alpha+W)$, which is given by left Kan extension along $s_{W}$. If $W$ is contained in $U$ up to $G$-orbits, then, by Theorem 1.2, the functors $s_{W}, s_{W}^{*}$, and $s_{*}^{W}$ are isomorphisms of categories.

(d) If $\iota: U \rightarrow U^{\prime}$ is a linear isometry, then there is a functor

$$
s_{\iota}: \mathfrak{B}_{G}^{U}(\alpha) \rightarrow \mathfrak{B}_{G}^{U^{\prime}}(\alpha)
$$

which is the identity on objects and which is given on morphisms by the composite

$$
\begin{aligned}
{\left[S_{U}^{\alpha} \wedge G / K_{+}, S_{U}^{\alpha} \wedge G / J_{+}\right]_{G}^{U} \stackrel{\iota *}{\longrightarrow}\left[\iota_{*}\left(S_{U}^{\alpha} \wedge G / K_{+}\right), \iota_{*}\left(S_{U}^{\alpha} \wedge G / J_{+}\right)\right]_{G}^{U^{\prime}} } \\
\\
\cong\left[S_{U^{\prime}}^{\alpha} \wedge G / K_{+}, S_{U^{\prime}}^{\alpha} \wedge G / J_{+}\right]_{G}^{U^{\prime}} .
\end{aligned}
$$

Precomposition with $s_{\iota}$ produces a functor $s_{\iota}^{*}: \mathcal{M}_{G}^{U^{\prime}}(\alpha) \rightarrow \mathcal{M}_{G}^{U}(\alpha)$. The functor $s_{\iota}^{*}$ has a left adjoint $s_{*}^{\iota}: \mathcal{M}_{G}^{U}(\alpha) \rightarrow \mathcal{M}_{G}^{U^{\prime}}(\alpha)$, which is given by left Kan extension along $s_{\iota}$. If $U$ and $U^{\prime}$ are $G$-orbit equivalent, then the functors $s_{\iota}, s_{\iota}^{*}$ and $s_{*}^{\iota}$ are isomorphisms of categories.

Remark 1.6. If $\alpha=0$, then $U$ - $\alpha$-Mackey functors are just $U$-Mackey functors in the sense of [13]. In particular, if $U$ is a complete $G$-universe and $\alpha=0$, then $U$ - $\alpha$-Mackey functors are just ordinary Mackey functors in the sense used in representation theory $[5,11,25]$. If $U$ is the trivial universe 
and $\alpha=0$, then $U$ - $\alpha$-Mackey functors are just Bredon-Illman coefficient systems $[1,10,28]$.

EXAMPLES 1.7. (a) If $Z$ is a $G$-spectrum indexed on the $G$-universe $U$ and $\alpha$ is an element of $R O^{+}(G, U)$, then the collection of homotopy groups $\pi_{\alpha}^{K, U} Z$, where $K$ runs over the subgroups of $G$, forms a $U$ - $\alpha$-Mackey functor $\underline{\pi}_{\alpha}^{G, U} Z$. The value of $\underline{\pi}_{\alpha}^{G, U} Z$ at the orbit $G / K$ is $\pi_{\alpha}^{K, U} Z=\left[S_{U}^{\alpha} \wedge G / K_{+}, Z\right]_{G}^{U}$. The effect of a morphism $f: S_{U}^{\alpha} \wedge G / J_{+} \rightarrow S_{U}^{\alpha} \wedge G / K_{+}$in $\mathfrak{B}_{G}^{U}(\alpha)$ is simply that of precomposition by $f$.

(b) If $Z, U$, and $\alpha$ are as in part (a) and $W$ is a finite-dimensional $G$-representation, then the maps $\sigma_{W}^{K}: \pi_{\alpha}^{K, U} Z \rightarrow \pi_{\alpha+W}^{K, U} \Sigma^{W} Z$ fit together to form a natural map $\sigma_{W}: \underline{\pi}_{\alpha}^{G, U} Z \rightarrow s_{W}^{*} \underline{\pi}_{\alpha+W}^{G, U} \Sigma^{W} Z$ describing the effect of suspension by $W$. Let $\widetilde{\sigma}_{W}: s_{*}^{W} \underline{\pi}_{\alpha}^{G, U} Z \rightarrow \underline{\pi}_{\alpha+W}^{G, U} \Sigma^{W} Z$ be the adjoint of $\sigma_{W}$ under the $\left(s_{*}^{W}, s_{W}^{*}\right)$-adjunction.

(c) Let $\iota: U \rightarrow U^{\prime}$ be a linear isometry between $G$-universes and let $Z$ be a $G$-spectrum indexed on $U$. Then the maps $\sigma_{\iota}^{K}: \pi_{\alpha}^{K, U} Z \rightarrow \pi_{\alpha}^{K, U^{\prime}} \iota_{*} Z$ fit together to form a natural map $\sigma_{\iota}: \underline{\pi}_{\alpha}^{G, U} Z \rightarrow s_{\iota}^{*} \underline{\pi}_{\alpha}^{G, U^{\prime}} \iota_{*} Z$ describing the effect of the change of universe functor $\iota_{*}$. Let $\widetilde{\sigma}_{\iota}: s_{*}^{\iota} \pi_{\alpha}^{G, U} Z \rightarrow \underline{\pi}_{\alpha}^{G, U^{\prime}} \iota_{*} Z$ be the adjoint of $\sigma_{\iota}$ under the $\left(s_{*}^{\iota}, s_{\iota}^{*}\right)$-adjunction.

The results in [12] on the space-level suspension map suggest that, when the functors $\Sigma^{W}$ and $\iota_{*}$ are not equivalences of categories, one should not expect the maps $\sigma_{W}: \underline{\pi}_{\alpha}^{G, U} Z \rightarrow s_{W}^{*} \underline{\pi}_{\alpha+W}^{G, U} \Sigma^{W} Z$ and $\sigma_{\iota}: \underline{\pi}_{\alpha}^{G, U} Z \rightarrow s_{\iota}^{*} \underline{\pi}_{\alpha}^{G, U^{\prime}} \iota_{*} Z$ to be well-behaved because the functors $\underline{\pi}_{\alpha+W}^{G, U} \Sigma^{W} Z$ and $\underline{\pi}_{\alpha}^{G, U^{\prime}} \iota_{*} Z$ carry a much richer structure than $\underline{\pi}_{\alpha}^{G, U} Z$. Instead, one should expect the adjoint maps $\widetilde{\sigma}_{W}$ and $\widetilde{\sigma}_{\iota}$ to be nicely behaved. Our second main result asserts that these adjoint maps are, in fact, isomorphisms on the bottom nonvanishing homotopy groups of $Z$ when $Z$ is a bounded-below spectrum.

Definition 1.8. A $G$-spectrum $Z$ indexed on $U$ is said to be $\alpha$-connected if $\underline{\pi}_{\alpha+n}^{G, U} Z=0$ for every integer $n \leq 0$. The full subcategory of $\widetilde{h} G \mathcal{S} U$ whose objects are the $(\alpha-1)$-connected spectra is denoted by $\widetilde{h} G \mathcal{S}(U, \alpha)$.

Theorem 1.9. Let $U$ be a $G$-universe, $\alpha$ be an element of $R O^{+}(G, U)$, and $Z$ be an $(\alpha-1)$-connected $G$-spectrum indexed on $U$. Then, for any finite-dimensional $G$-representation $W$ and any linear isometry $\iota: U \rightarrow U^{\prime}$, the maps

$$
\widetilde{\sigma}_{W}: s_{*}^{W} \underline{\pi}_{\alpha}^{G, U} Z \rightarrow \underline{\pi}_{\alpha+W}^{G, U} \Sigma^{W} Z \quad \text { and } \quad \widetilde{\sigma}_{\iota}: s_{*}^{\iota} \underline{\pi}_{\alpha}^{G, U} Z \rightarrow \underline{\pi}_{\alpha}^{G, U^{\prime}} \iota_{*} Z
$$

are isomorphisms.

Remark 1.10. The assertion, in Theorem 1.9, that the map $\widetilde{\sigma}_{\iota}$ is an isomorphism when $Z$ is an $(\alpha-1)$-connected spectrum generalizes Proposition 6.1 in [13], which applies only to the case $\alpha=0$. 
Theorem 1.9 suffers from the same defect as that suffered by the spacelevel equivariant suspension theorem presented in [12]; that is, it describes the behavior of the functors $\Sigma^{W}$ and $\iota_{*}$ only on the lowest-dimensional nonvanishing homotopy groups. Moreover, the requirement in this theorem that $Z$ be bounded below is quite restrictive. Our third main result gives two spectral sequences that provide a description of the maps $\widetilde{\sigma}_{W}$ and $\widetilde{\sigma}_{\iota}$ and the homotopy functors $\underline{\pi}_{\alpha+W}^{G, U} \Sigma^{W} Z$ and $\underline{\pi}_{\alpha}^{G, U^{\prime}} \iota_{*} Z$ that is applicable to an arbitrary $G$-spectrum $Z$ indexed on an arbitrary $G$-universe $U$. These spectral sequences are derived from a Postnikov tower decomposition of the spectrum $Z$. To describe their $E^{2}$-terms, we must introduce the sort of equivariant Eilenberg-MacLane spectra that are used to form this Postnikov tower.

Definition 1.11. If $M$ is a $U$ - $\alpha$-Mackey functor, then a $U$ - $\alpha$-EilenbergMacLane spectrum $K_{U}^{G}(M, \alpha)$ is a $G$-spectrum indexed on $U$ with the $G$-homotopy type of a $G$-CW spectrum such that $\underline{\pi}_{\alpha}^{G, U} K_{U}^{G}(M, \alpha)=M$ and $\underline{\pi}_{\alpha+n}^{G, U} K_{U}^{G}(M, \alpha)=0$ for every nonzero integer $n$. Note that a $U$ - $\alpha$-EilenbergMacLane spectrum is $(\alpha-1)$-connected by definition.

Remark 1.12. If $U$ is a complete $G$-universe, $\alpha=0$, and $M$ is a $U$ - $\alpha$ Mackey functor, then the spectrum $K_{U}^{G}(M, \alpha)$ represents $R O(G)$-graded equivariant ordinary cohomology with $M$ coefficients as defined in [17]. If $U$ is not complete, but $\alpha=0$, then $K_{U}^{G}(M, \alpha)$ represents the cohomology theory that should be thought of as the appropriate analog for the universe $U$ of $R O(G)$-graded equivariant ordinary cohomology. This cohomology theory is graded on $R O^{+}(G, U)$ and satisfies the obvious dimension axiom.

The following result, which is proved in Section 3, assures us that $U-\alpha-$ Eilenberg-MacLane spectra exist and behave as they ought to.

TheOREM 1.13. For each $U$ - $\alpha$-Mackey functor $M$, there is a $U$ - $\alpha$ Eilenberg-MacLane spectrum $K_{U}^{G}(M, \alpha)$ in $G \mathcal{S U}$; this spectrum is unique up to G-homotopy equivalence. Moreover, the assignment of $K_{U}^{G}(M, \alpha)$ to $M$ gives a functor from $\mathcal{M}_{G}^{U}(\alpha)$ to $\widetilde{h} G \mathcal{S}(U, \alpha)$ which is right adjoint to the functor $\underline{\pi}_{\alpha}^{G, U}: \widetilde{h} G \mathcal{S}(U, \alpha) \rightarrow \mathcal{M}_{G}^{U}(\alpha)$. The counit $\varepsilon_{\alpha}^{U}: \underline{\pi}_{\alpha}^{G, U} K_{U}^{G}(M, \alpha) \rightarrow M$ of this adjunction is an isomorphism identifying $\underline{\pi}_{\alpha}^{G, U} K_{U}^{G}(M, \alpha)$ with $M$.

Essentially, our two spectral sequences reduce the problem of understanding the behavior of the functors $\Sigma^{W}$ and $\iota_{*}$ on arbitrary spectra in $\widetilde{h} G \mathcal{S} U$ to the problem of understanding their behavior on EilenbergMacLane spectra.

Theorem 1.14. Let $Z$ be a $G$-spectrum indexed on the $G$-universe $U$. Then 
(a) For each finite-dimensional $G$-representation $W$, there is a right halfplane spectral sequence whose $E^{2}$-term is given by

$$
E_{p, q}^{2}=\underline{\pi}_{\alpha+W+p+q}^{G, U}\left(\Sigma^{W} K_{U}^{G}\left(\underline{\pi}_{\alpha+q}^{G, U} Z, \alpha+q\right)\right) .
$$

This spectral sequence is natural in $Z$ and converges in total degree $n$ to $\underline{\pi}_{\alpha+W+n}^{G, U} \Sigma^{W} Z$. Moreover, for any integer $q, E_{0, q}^{2} \cong s_{*}^{W} \underline{\pi}_{\alpha+q}^{G, U} Z$. Under this isomorphism, the edge homomorphism

$$
E_{0, q}^{2} \rightarrow E_{0, q}^{\infty} \subset \underline{\pi}_{\alpha+W+q}^{G, U} \Sigma^{W} Z
$$

is identified with the map $\widetilde{\sigma}_{W}: s_{*}^{W} \underline{\pi}_{\alpha+q}^{G, U} Z \rightarrow \underline{\pi}_{\alpha+W+q}^{G, U} \Sigma^{W} Z$ of Example $1.7(b)$.

(b) If $\iota: U \rightarrow U^{\prime}$ is a linear isometry, then there is a right half-plane spectral sequence whose $E^{2}$-term is given by

$$
E_{p, q}^{2}=\underline{\pi}_{\alpha+p+q}^{G, U^{\prime}}\left(\iota_{*} K_{U}^{G}\left(\underline{\pi}_{\alpha+q}^{G, U} Z, \alpha+q\right)\right) .
$$

This spectral sequence is natural in $Z$ and converges in total degree $n$ to $\underline{\pi}_{\alpha+n}^{G, U^{\prime}} \iota_{*} Z$. Moreover, for any integer $q, E_{0, q}^{2} \cong s_{*}^{\iota} \underline{\pi}_{\alpha+q}^{G, U} Z$. Under this isomorphism, the edge homomorphism

$$
E_{0, q}^{2} \rightarrow E_{0, q}^{\infty} \subset \underline{\pi}_{\alpha+q}^{G, U^{\prime}} \iota_{*} Z
$$

is identified with the map $\widetilde{\sigma}_{\iota}: s_{*}^{\iota} \underline{\pi}_{\alpha+q}^{G, U} Z \rightarrow \underline{\pi}_{\alpha+q}^{G, U^{\prime}} \iota_{*} Z$ of Example 1.7(c).

Remarks 1.15. (a) The spectral sequence of part (a) of the theorem converges to $\underline{\pi}_{\alpha+W+n}^{G, U} \Sigma^{W} Z$ in the usual sense that there is a filtration $\left\{F_{p} \underline{\pi}_{\alpha+W+n}^{G, U} \Sigma^{W} Z\right\}$ of $\underline{\pi}_{\alpha+W+n}^{G, U} \Sigma^{W} Z$ with the three properties that $F_{p} \underline{\pi}_{\alpha+W+n}^{G, U} \Sigma^{W} Z=0$, for $p<0$, that $\underline{\pi}_{\alpha+W+n}^{G, U} \Sigma^{W} Z=\bigcup_{p} F_{p} \underline{\pi}_{\alpha+W+n}^{G, U} \Sigma^{W} Z$, and that

$$
E_{p, q}^{\infty} \cong F_{p} \underline{\pi}_{\alpha+p+q}^{G, U} \Sigma^{W} Z / F_{p-1} \underline{\pi}_{\alpha+p+q}^{G, U} \Sigma^{W} Z
$$

The spectral sequence of part (b) converges in the analogous sense.

(b) The spectral sequences of the theorem are spectral sequences of $U$ - $(\alpha+W)$-Mackey functors and $U^{\prime}$ - $\alpha$-Mackey functors, respectively. However, by taking the values of these generalized Mackey functors at an orbit $G / K$, we obtain spectral sequences of abelian groups which converge to the abelian groups $\pi_{\alpha+W+p+q}^{K, U} \Sigma^{W} Z$ and $\pi_{\alpha+p+q}^{K, U^{\prime}} Z$. The advantage of the generalized Mackey functor versions of these spectral sequences is that, in this form, one can use restriction and transfer maps as an aid to computing differentials. Without using these Mackey functor structure maps, we are not likely to be able to do many serious calculations with these spectral sequences.

(c) If $Z$ is $(\alpha-1)$-connected, then these two spectral sequences become first quadrant spectral sequences. In this case, for each of the spectral sequences, the only nonvanishing $E^{2}$-entry in total degree 0 is $E_{0,0}^{2}$. This entry 
survives unchanged to $E^{\infty}$. Thus, for $q=0$, the edge homomorphisms of the two spectral sequences are isomorphisms. These are precisely the isomorphisms described in Theorem 1.9. Thus, Theorem 1.14 is a generalization of Theorem 1.9.

(d) Both of the spectral sequences in the theorem above are homology versions of the cohomology spectral sequences of type (B.3) discussed by Greenlees and May in Appendix B of [6].

Having cluttered algebraic topology with yet two more spectral sequences, it seems only proper to address the question of the computability of their $E^{2}$-terms. For the spectral sequence of part (b), a reasonably positive answer to this question can be given in some important special cases. When $\alpha=0$ and $U$ is the trivial universe, the splitting theorem of [15] provides an alternative description of $\underline{\pi}_{\alpha+n}^{G, U^{\prime}} \iota_{*} Z$ for any $G$-spectrum $Z$ indexed on $U$. This description need not be terribly enlightening when $Z$ is an arbitrary $G$-spectrum. However, applied to Eilenberg-MacLane spectra, this splitting gives a complete description of our $E^{2}$-term. If $G$ is a finite group, this description is in terms of ordinary group homology.

Proposition 1.16. Let $G$ be a finite group, $\iota: U \rightarrow U^{\prime}$ be the inclusion of a trivial $G$-universe $U$ into an arbitrary $G$-universe $U^{\prime}, Z$ be a $G$-spectrum indexed on $U$, and $\alpha \in R O^{+}(G, U)$ be 0 . Then, for any subgroup $H$ of $G$, the value of the $U^{\prime}-\alpha$-Mackey functor $E^{2}$-term of the spectral sequence of Theorem 1.14(b) at $G / H$ is given by

$$
E_{p, q}^{2}(G / H)=\bigoplus_{(K)_{H}} H_{p}\left(W_{H} K ; \pi_{q}^{K} Z\right),
$$

where the sum runs over the $H$-conjugacy classes $(K)_{H}$ of those subgroups $K$ of $H$ such that $H / K H$-imbeds in $U^{\prime}$. Here, $W_{H} K$ is the Weyl group of the subgroup $K$, which acts on $\pi_{q}^{K} Z \cong \pi_{q}\left(Z^{K}\right)$ via the usual action of $W_{H} K$ on the fixed-point subspectrum $Z^{K}$ of $Z$.

Remarks 1.17. (a) The splitting from [15] which forms the basis for this proposition comes from a natural filtration of $Z$ derived from the poset of subgroups of $H$ (see [15]). So long as $G$ is finite, this filtration can be used to study the $E^{2}$-terms of both of the spectral sequences of Theorem 1.14 for any $\alpha, U, W$, and $\iota: U \rightarrow U^{\prime}$.

(b) The splitting in [15] is a generalization of one of the splittings introduced in [9]. There are other splittings in [9] related to the space-level suspension functor $\Sigma^{W}$. This suggests that there might be a splitting of the homotopy groups of a spectrum of the form $\Sigma^{W} Z$ which could be used to compute the $E^{2}$-term of the spectral sequence of part (a) of the theorem in some special cases. 
(c) The homotopy functors which appear in the $E^{2}$-term of the spectral sequence of part (a) of the theorem are all parts of the $\mathrm{RO}^{+}(G, U)$-graded equivariant ordinary cohomology of a point. If $W$ is very low-dimensional, then it may be possible to use the geometry of $W$ to compute these functors. See the appendix of [14] for some examples of the ways in which the geometry of representations can be used to compute the $\mathrm{RO}^{+}(G, U)$-graded equivariant ordinary cohomology of a point.

If the sequence

$$
0 \rightarrow M^{\prime} \rightarrow M \rightarrow M^{\prime \prime} \rightarrow 0
$$

is a short exact sequence of $U$ - $\alpha$-Mackey functors, then the sequence

$$
K_{U}^{G}\left(M^{\prime}, \alpha\right) \rightarrow K_{U}^{G}(M, \alpha) \rightarrow K_{U}^{G}\left(M^{\prime \prime}, \alpha\right)
$$

is a fibre sequence in $\widetilde{h} G \mathcal{S} U$. The functors $\Sigma^{W}$ and $\iota_{*}$ preserve fibre sequences. Thus, the above short exact sequence of $U$ - $\alpha$-Mackey functors gives rise to two long exact sequences of the form

$$
\begin{aligned}
\ldots \rightarrow \underline{\pi}_{\alpha+1}^{G, U^{\prime}} \iota_{*} K_{U}^{G}(M, \alpha) & \rightarrow \underline{\pi}_{\alpha+1}^{G, U^{\prime}} \iota_{*} K_{U}^{G}\left(M^{\prime \prime}, \alpha\right) \\
& \rightarrow s_{*}^{\iota} M^{\prime} \rightarrow s_{*}^{\iota} M \rightarrow s_{*}^{\iota} M^{\prime \prime} \rightarrow 0
\end{aligned}
$$

and

$$
\begin{aligned}
\ldots \rightarrow \underline{\pi}_{\alpha+W+1}^{G, U} \Sigma^{W} K_{U}^{G}(M, \alpha) & \rightarrow \underline{\pi}_{\alpha+W+1}^{G, U} \Sigma^{W} K_{U}^{G}\left(M^{\prime \prime}, \alpha\right) \\
& \rightarrow s_{*}^{W} M^{\prime} \rightarrow s_{*}^{W} M \rightarrow s_{*}^{W} M^{\prime \prime} \rightarrow 0 .
\end{aligned}
$$

It is therefore tempting to assume that the functors $\underline{\pi}_{\alpha+n}^{G, U^{\prime}} \iota_{*} K_{U}^{G}(?, \alpha)$ and $\underline{\pi}_{\alpha+W+n}^{G, U} \Sigma^{W} K_{U}^{G}(?, \alpha)$, for $n \geq 1$, are the derived functors of $s_{*}^{\iota}$ and $s_{*}^{W}$, respectively. This conjecture, if it were true, would give a very attractive algebraic description of the $E^{2}$-terms of the spectral sequences of Theorem 1.14. In [12], it was suggested that this conjecture was true. However, if this conjecture were true, then the functors $\underline{\pi}_{\alpha+n}^{G, U^{\prime}} \iota_{*} K_{U}^{G}(?, \alpha)$ and $\underline{\pi}_{\alpha+W+n}^{G, U} \Sigma^{W} K_{U}^{G}(?, \alpha)$, for $n \geq 1$, would have to vanish on projective $U$ - $\alpha$ Mackey functors. Since the category $\mathcal{M}_{G}^{U}(\alpha)$ is a functor category, its projectives are direct summands of direct sums of the representable $U$ - $\alpha$-Mackey functors $\underline{\pi}_{\alpha}^{G, U}\left(S_{U}^{\alpha} \wedge G / K_{+}\right)$, for $K \leq G$. Thus, the question of whether or not $\underline{\pi}_{\alpha+n}^{G, U^{\prime}} \iota_{*} K_{U}^{G}(?, \alpha)$ and $\underline{\pi}_{\alpha+W+n}^{G, U} \Sigma^{W} K_{U}^{G}(?, \alpha)$ are derived functors reduces to the question of whether or not they vanish on the representable functors $\underline{\pi}_{\alpha}^{G, U}\left(S_{U}^{\alpha} \wedge G / K_{+}\right)$. In fact, as the following example indicates, they need not vanish on representable functors and are therefore not the derived functors.

EXAMPLE 1.18. Let $G=\mathbb{Z} / p$, where $p$ is a prime, $\iota: U \rightarrow U^{\prime}$ be the inclusion of a trivial $G$-universe $U$ into a complete $G$-universe $U^{\prime}, \alpha=0$, and $M$ be the representable $U$-0-Mackey functor $\underline{\pi}_{0}^{G, U}\left(S_{U}^{0}\right)$. Since $U$ is a 
trivial $G$-universe, a $U$-0-Mackey functor is just a Bredon coefficient system. The representable coefficient system $\underline{\pi}_{0}^{G, U}\left(S_{U}^{0}\right)$ is easily seen to be the constant coefficient system at $\mathbb{Z}$. By the splitting theorem of [15], if $n>0$, then the value of the $U^{\prime}$-0-Mackey functor $\underline{\pi}_{n}^{G, U^{\prime}} \iota_{*} K_{U}^{G}(M, 0)$ at $G / G$ is just $H_{n}(\mathbb{Z} / p ; \mathbb{Z})$, which need not be zero. Thus, the functor $\underline{\pi}_{n}^{G, U^{\prime}} \iota_{*} K_{U}^{G}(?, 0)$ does not vanish on the projective $U$-0-Mackey functor $M=\underline{\pi}_{0}^{G, U}\left(S_{U}^{0}\right)$, for $n>0$, and the functors $\underline{\pi}_{\alpha+n}^{G, U^{\prime}} \iota_{*} K_{U}^{G}(?, \alpha)$ are not the derived functors of $s_{*}^{\iota}$ in this case. Using this same $G, U, \alpha$ and a sufficiently large nontrivial $G$-representation $W$, one easily obtains an example in which the functors $\underline{\pi}_{\alpha+W+n}^{G, U} \Sigma^{W} K_{U}^{G}(?, \alpha)$ are not the derived functors of $s_{*}^{W}$. The assertion in [12] that the $E^{2}$-term of the spectral sequence mentioned there was given by the derived functors was a mis-statement caused by a miscalculation.

Two other questions that arise naturally when a new spectral sequence is introduced are whether the sequence must always collapse and whether nontrivial extensions arise in recovering the target of the spectral sequence from the $E^{\infty}$-term. We conclude this section with a sample calculation using the spectral sequence of part (b) of Theorem 1.14 which shows that this spectral sequence need not collapse and that nontrivial extensions may occur in recovering the target. This example also illustrates the way in which the Mackey functor structure of the spectral sequences can be used to facilitate computations. An analogous example using the same $G, \alpha, U$, and $Z$, and a sufficiently large $G$-representation $W$ shows that the spectral sequence of part (a) of the theorem need not collapse and may give rise to nontrivial extensions in recovering its target. Our example is a stable version of the examples used in [13] and [12] to illustrate the misbehavior of the equivariant Hurewicz and suspension maps.

Throughout the remainder of this section, $G$ is the cyclic group $\mathbb{Z} / m$, for some odd prime $m, \iota: U \rightarrow U^{\prime}$ is the inclusion of a trivial $G$-universe $U$ into a complete $G$-universe $U^{\prime}, \alpha=0$, and $V$ is a nontrivial irreducible complex $G$-representation. Take $Z$ to be the $G$-spectrum $\Sigma_{U}^{\infty} S V_{+}$indexed on $U$; then $\iota_{*} Z$ is $\Sigma_{U^{\prime}}^{\infty} S V_{+}$. Theorem $1.14(\mathrm{~b})$ provides a spectral sequence converging to the homotopy functors $\underline{\pi}_{n}^{G, U^{\prime}} \Sigma_{U^{\prime}}^{\infty} S V_{+}$whose $E^{2}$-term is determined by the functors $\underline{\pi}_{n}^{G, U} \Sigma_{U}^{\infty} S V_{+}$. We want to investigate the behavior of this spectral sequence in total degree $n \leq 2$. We begin this investigation by computing both the $E^{2}$-term of the spectral sequence (in terms of the nonequivariant stable stems) and the target functor $\underline{\pi}_{n}^{G, U^{\prime}} \Sigma_{U^{\prime}}^{\infty} S V_{+}$ for $n \leq 2$. Note that $U$-0-Mackey functors and $U^{\prime}-0$-Mackey functors are just Bredon coefficient systems and Mackey functors, respectively, for the group $G$. A few observations about these types of functors, drawn mostly from the first section and the appendix of [14], provide some essential background for our calculations. To describe a Bredon coefficient system $M$ for 
the group $G$, one must specify the abelian groups $M(G / G)$ and $M(G / e)$ which are the values of $M$ at the orbits $G / G$ and $G / e$, the restriction map $r: M(G / G) \rightarrow M(G / e)$, and the action of $G$ on $M(G / e)$. The only constraint on this data is that the image of $r$ must lie in the subgroup $M(G / e)^{G}$ of $G$-invariant elements of $M(G / e)$. To describe a Mackey functor $N$ for the group $G$, one must specify the values $N(G / G)$ and $N(G / e)$ of $N$, the restriction map $r: N(G / G) \rightarrow N(G / e)$, the transfer map $t: N(G / e) \rightarrow N(G / G)$, and the action of $G$ on $N(G / e)$. The constraints on this data are that the image of $r$ must lie in $N(G / e)^{G}$, the transfer map $t$ must factor through the projection $N(G / e) \rightarrow N(G / e) / G$, and the composite $r \circ t$ must equal the trace map of the action of $G$ on $N(G / e)$. Note that, if the action of $G$ on $N(G / e)$ is trivial, then the composite $r \circ t$ is just multiplication by $m$. There are three commonly occurring Mackey functors, $L(A), R(A)$, and $A / / 0$, and one commonly occurring coefficient system $0 / / A$ associated with each abelian group $A$. The values of $L(A)$ and $R(A)$ at both $G / G$ and $G / e$ are just $A$. The transfer map in $L(A)$ and the restriction map in $R(A)$ are both the identity map. The restriction map in $L(A)$ and the transfer map in $R(A)$ are both multiplication by $m$. The group $A$ is the value of $A / / 0$ at $G / G$ and of $0 / / A$ at $G / e$. The trivial group is the value of $A / / 0$ at $G / e$ and of $0 / / A$ at $G / G$. The restriction maps in $A / / 0$ and $0 / / A$ and the transfer map in $A / / 0$ are all zero. In all four functors, $G$ acts trivially on the value at $G / e$. Both the $E^{2}$-term of the spectral sequence in our example and its target $\underline{\pi}_{n}^{G, U^{\prime}} \Sigma_{U^{\prime}}^{\infty} S V_{+}$can be described in terms of these four special types of Mackey functors and coefficient systems.

Lemma 1.19. (a) For any integer $q$,

$$
\underline{\pi}_{q}^{G, U} \Sigma_{U}^{\infty} S V_{+} \cong 0 / /\left(\pi_{q} S^{0} \oplus \pi_{q-1} S^{0}\right)
$$

where the groups $\pi_{q} S^{0}$ and $\pi_{q-1} S^{0}$ are the nonequivariant stable stems.

(b) For any integer $q$,

$$
E_{0, q}^{2}=L\left(\pi_{q} S^{0} \oplus \pi_{q-1} S^{0}\right),
$$

where again the groups $\pi_{q} S^{0}$ and $\pi_{q-1} S^{0}$ are the nonequivariant stable stems.

(c) For any integers $p$ and $q$ with $p>0$,

$$
E_{p, q}^{2}=H_{p}\left(G ; \pi_{q} S^{0} \oplus \pi_{q-1} S^{0}\right) / / 0 .
$$

Proof. Part (a) follows from the freeness of the action of $G$ on $S V$, the triviality of the universe $U$, and the nonequivariant splitting $\Sigma_{U}^{\infty} S V_{+} \cong$ $S_{U}^{0} \vee S_{U}^{1}$. The triviality of the action of $G$ on the value of $\underline{\pi}_{q}^{G, U} \Sigma_{U}^{\infty} S V_{+}$at $G / e$ follows from the fact that $G$ acts homotopically trivially on $S V_{+}$. Part (b) follows from part (a) and either the description of the functor $s_{*}^{\iota}$ in [13] or Proposition 1.16. Part (c) also follows from part (a) and Proposition 1.16. 
Lemma 1.20. For $G, U^{\prime}$, and $V$ as defined above,

$$
\begin{aligned}
\underline{\pi}_{0}^{G, U^{\prime}} \Sigma_{U^{\prime}}^{\infty} S V_{+} & =L(\mathbb{Z}), \\
\underline{\pi}_{1}^{G, U^{\prime}} \Sigma_{U^{\prime}}^{\infty} S V_{+} & =L(\mathbb{Z} / 2) \oplus R(\mathbb{Z}) \cong R(\mathbb{Z} / 2) \oplus R(\mathbb{Z}) \cong R(\mathbb{Z} / 2 \oplus \mathbb{Z}), \\
\underline{\pi}_{2}^{G, U^{\prime}} \Sigma_{U^{\prime}}^{\infty} S V_{+} & =L(\mathbb{Z} / 2) \oplus R(\mathbb{Z} / 2) \cong L(\mathbb{Z} / 2) \oplus L(\mathbb{Z} / 2) \\
& \cong L(\mathbb{Z} / 2 \oplus \mathbb{Z} / 2) .
\end{aligned}
$$

P r o of. We make use of the techniques developed in the appendix to [14]. As in the proof of Lemma A.1 of [14], for each integer $q$, the standard cofibre sequence

$$
G_{+} \rightarrow S V_{+} \rightarrow \Sigma G_{+}
$$

yields a short exact sequence of Mackey functors

$$
0 \rightarrow L\left(\pi_{q} S^{0}\right) \rightarrow \underline{\pi}_{q}^{G, U^{\prime}} \Sigma_{U^{\prime}}^{\infty} S V_{+} \rightarrow R\left(\pi_{q-1} S^{0}\right) \rightarrow 0,
$$

where $\pi_{q} S^{0}$ and $\pi_{q-1} S^{0}$ are the nonequivariant stable stems. The first isomorphism of the lemma follows immediately from this exact sequence and the vanishing of $\pi_{-1} S^{0}$. To obtain the second and third isomorphisms, we must show that our short exact sequence of Mackey functors splits for $q=1$ and 2. If $q=1$, then when evaluated at either $G / G$ or $G / e$, the short exact sequence must split since the two values of $R(\mathbb{Z})$ are both $\mathbb{Z}$. Valuewise splittings like this do not usually imply that the sequence splits as a short exact sequence of Mackey functors. However, it is fairly easy to use the restriction and transfer maps of $\pi_{1}^{G, U^{\prime}} \Sigma_{U^{\prime}}^{\infty} S V_{+}$to argue that the Mackey functor short exact sequence does in fact split for $q=1$. Thus, $\underline{\pi}_{1}^{G, U^{\prime}} \Sigma_{U^{\prime}}^{\infty} S V_{+} \cong L(\mathbb{Z} / 2) \oplus R(\mathbb{Z})$. Since multiplication by $m$ is an isomorphism on $\mathbb{Z} / 2, L(\mathbb{Z} / 2)$ and $R(\mathbb{Z} / 2)$ are isomorphic. The remaining isomorphisms identifying $\underline{\pi}_{1}^{G, U^{\prime}} \Sigma_{U^{\prime}}^{\infty} S V_{+}$are derived from this isomorphism. The value of $\underline{\pi}_{2}^{G, U^{\prime}} \Sigma_{U^{\prime}}^{\infty} S V_{+}$at $G / e$ is the nonequivariant stable homotopy group $\pi_{2} S V_{+}$, which is $\mathbb{Z} / 2 \oplus \mathbb{Z} / 2$. The composite $r \circ t$ in $\underline{\pi}_{2}^{G, U^{\prime}} \Sigma_{U^{\prime}}^{\infty} S V_{+}$ is multiplication by the odd prime $m$ and is therefore an isomorphism. From this and the short exact sequence, it follows immediately that the value of $\underline{\pi}_{2}^{G, U^{\prime}} \Sigma_{U^{\prime}}^{\infty} S V_{+}$at $G / G$ must also be $\mathbb{Z} / 2 \oplus \mathbb{Z} / 2$. Once the value of $\underline{\pi}_{2}^{G, U^{\prime}} \Sigma_{U^{\prime}}^{\infty} S V_{+}$at $G / G$ is known, arguments with the restriction and transfer maps of $\underline{\pi}_{2}^{G, U^{\prime}} \Sigma_{U^{\prime}}^{\infty} S V_{+}$, like those used for $\underline{\pi}_{1}^{G, U^{\prime}} \Sigma_{U^{\prime}}^{\infty} S V_{+}$, make it clear that the Mackey functor short exact sequence also splits when $q=2$. Thus, $\underline{\pi}_{2}^{G, U^{\prime}} \Sigma_{U^{\prime}}^{\infty} S V_{+} \cong L(\mathbb{Z} / 2) \oplus R(\mathbb{Z} / 2)$. The remaining isomorphisms identifying $\underline{\pi}_{2}^{G, U^{\prime}} \Sigma_{U^{\prime}}^{\infty} S V_{+}$follow from the isomorphism between $L(\mathbb{Z} / 2)$ and $R(\mathbb{Z} / 2)$ described above.

We can now address our questions about collapsing and nontrivial extensions. For the question about nontrivial extensions, note that, in our 
example, $E^{2}$ must equal $E^{\infty}$ in total degree 1 for dimensional reasons. The short exact sequence description

$$
0 \rightarrow E_{0,1}^{\infty} \rightarrow \underline{\pi}_{1}^{G, U^{\prime}} \Sigma_{U^{\prime}}^{\infty} S V_{+} \rightarrow E_{1,0}^{\infty} \rightarrow 0
$$

of $\underline{\pi}_{1}^{G, U^{\prime}} \Sigma_{U^{\prime}}^{\infty} S V_{+}$coming from the two nonvanishing $E^{\infty}$-terms in total degree 1 therefore has the form

$$
0 \rightarrow L(\mathbb{Z} \oplus \mathbb{Z} / 2) \rightarrow R(\mathbb{Z} \oplus \mathbb{Z} / 2) \rightarrow(\mathbb{Z} / m) / / 0 \rightarrow 0 .
$$

There is a unique map of Mackey functors from $L(\mathbb{Z} \oplus \mathbb{Z} / 2)$ to $R(\mathbb{Z} \oplus \mathbb{Z} / 2)$ which is, as the map above must be, the identity when evaluated at $G / e$. Simple arguments with the restriction and transfer maps then indicate that, evaluated at $G / G$, the short exact sequence of Mackey functors above must be the nonsplit short exact sequence

$$
0 \rightarrow \mathbb{Z} \oplus \mathbb{Z} / 2 \stackrel{m \oplus 1}{\longrightarrow} \mathbb{Z} \oplus \mathbb{Z} / 2 \rightarrow \mathbb{Z} / m \rightarrow 0
$$

of abelian groups. Thus, nontrivial extension problems can occur in recovering our target homotopy groups from the $E^{\infty}$-term of our spectral sequence.

For the question about nontrivial differentials, note that, in this example, the only nonvanishing $E^{2}$-terms in total degree 2 are $E_{0,2}^{2}=L(\mathbb{Z} / 2 \oplus \mathbb{Z} / 2)$ and $E_{1,1}^{2}=(\mathbb{Z} / m) / / 0$. Since there is no $m$-torsion in

$$
\underline{\pi}_{2}^{G, U^{\prime}} \Sigma_{U^{\prime}}^{\infty} S V_{+} \cong L(\mathbb{Z} / 2 \oplus \mathbb{Z} / 2)
$$

the copy of $\mathbb{Z} / m$ in $E_{1,1}^{2}$ cannot survive to $E^{\infty}$. It follows for dimensional reasons that

$$
d^{2}: E_{3,0}^{2} \rightarrow E_{1,1}^{2}
$$

must be an isomorphism. Thus, there can be nontrivial differentials in our spectral sequences.

2. The suspension spectral sequence and the space-level suspension map. In this section, we relate the spectral sequence of Theorem 1.14(a) to the discussion of the space-level equivariant suspension map in [12]. The unstable equivariant suspension theorem (Theorem 2.5) in [12] is an unstable analog of Theorem 1.9. Let $V$ and $W$ be $G$-representations with $\left|V^{G}\right| \geq 1$, and let $Y$ be a based $G$-space. Theorem 2.5 of [12] asserts that, if $Y$ is $(V-1)$-connected (that is, if $\left|V^{K}\right|-1$ is less than or equal to the connectivity $c\left(Y^{K}\right)$ of $Y^{K}$ for every subgroup $K$ of $\left.G\right)$, then there is an isomorphism

$$
\widetilde{\sigma}: s_{*} \underline{\pi}_{V}^{G} Y \rightarrow \underline{\pi}_{V+W}^{G} \Sigma^{W} Y
$$

relating the $(V+W)$ th unstable homotopy functor $\pi_{V+W}^{G} \Sigma^{W} Y$ of $\Sigma^{W} Y$ to the $V$ th unstable homotopy functor $\pi_{V}^{G} Y$ of $Y$. The functor $s_{*}$ in this isomorphism is an unstable analog of the functor $s_{*}^{W}$ of Theorem 1.9. If $\left|V^{G}\right|=1$, then one cannot expect to be able to derive $\underline{\pi}_{V+W}^{G} \Sigma^{W} Y$ from $\underline{\pi}_{V}^{G} Y$ unless $Y$ 
is $(V-1)$-connected. However, if $\left|V^{G}\right| \geq 2$, then the nonequivariant suspension theorem would suggest that it might be possible to obtain $\pi_{V+W}^{G} \Sigma^{W} Y$ from $\underline{\pi}_{V}^{G} Y$ whenever $\left|V^{K}\right| \leq 2 c\left(Y^{K}\right)$, for all $K \leq G$. In fact, the value of $\underline{\pi}_{V}^{G} Y$ alone is not sufficient for the computation of $\underline{\pi}_{V+W}^{G}{ }^{W} Y$. Nevertheless, Theorem 2.5 of [12] does extend to a result which provides an unstable suspension spectral sequence describing the effect of suspension by $W$ in precisely the range of dimensions one would expect based on the nonequivariant suspension theorem. The relation between Theorem 2.5 of [12] and this extension is the same as the relation between Theorem 1.9 and its extension, Theorem 1.14. The portion of the $E^{2}$-term of the unstable suspension spectral sequence that is relevant for the computation of $\pi_{V+W}^{G} \Sigma^{W} Y$ is determined by the unstable homotopy functors $\underline{\pi}_{V}^{G} \Sigma^{-q} Y$ for $q \leq 0$ (the unusual sign convention on $q$ is picked to conform with the grading in the spectral sequence).

Our unstable suspension spectral sequence is derived from a special case of the stable suspension spectral sequence of Theorem 1.14(a) by identifying some of the stable homotopy functors appearing in the stable spectral sequence with analogous unstable homotopy functors. In particular, the unstable homotopy functors $\underline{\pi}_{V}^{G} \Sigma^{-q} Y$ are identified with the stable homotopy functors $\underline{\pi}_{V+q}^{G, U} \Sigma_{U}^{\infty} Y$ computed in the trivial $G$-universe $U=\mathbb{R}^{\infty}$. This identification implies that, associated with the unstable homotopy functors $\underline{\pi}_{V}^{G} \Sigma^{-q} Y$, there are equivariant Eilenberg-MacLane spectra $K_{U}^{G}\left(\underline{\pi}_{V}^{G} \Sigma^{-q} Y, V+q\right)$ indexed on $U$. The homotopy functors $\underline{\pi}_{V}^{G} \Sigma^{-q} Y$ determine the $E^{2}$-term of our unstable suspension spectral sequence in the sense that this $E^{2}$-term is made up of certain equivariant stable homotopy groups of the spectra $K_{U}^{G}\left(\underline{\pi}_{V}^{G} \Sigma^{-q} Y, V+q\right)$. Here, the discussion immediately preceding Example 1.18 should be recalled. It would have been nice to have a description of the $E^{2}$-term of the unstable suspension spectral sequence in terms of the functor $s_{*}$ mentioned above and its derived functors. Example 1.18 indicates that such a description is not possible. The equivariant stable homotopy groups of the spectra $K_{U}^{G}\left(\underline{\pi}_{V}^{G} \Sigma^{-q} Y, V+q\right)$ should be thought of as an approximation to these derived functors.

In order to describe our unstable suspension spectral sequence precisely and to derive it from Theorem 1.14(a), we must review some of the basic ideas from [12] and [13], including the definitions of the unstable homotopy functors $\underline{\pi}_{V}^{G} Y$ and $\underline{\pi}_{V+W}^{G}{ }^{W} Y$. Assume hereafter that $V$ is a $G$-representation with $\left|V^{G}\right| \geq 2$. Recall, from [13], that the category $\mathfrak{B}_{G}(V)$ has, as its objects, the orbits $G / K$ of $G$. The set of morphisms in $\mathfrak{B}_{G}(V)$ from the orbit $G / J$ to the orbit $G / K$ is $\left[\Sigma^{V} G / J_{+}, \Sigma^{V} G / K_{+}\right]_{G}$. A $V$-Mackey functor is a contravariant additive functor from $\mathfrak{B}_{G}(V)$ to the category $A b$ of abelian groups. The category of all $V$-Mackey functors is denoted by $\mathcal{M}_{G}(V)$. If $W$ is any other finite-dimensional representation, then suspen- 
sion by $W$ gives a functor

$$
s_{W}: \mathfrak{B}_{G}(V) \rightarrow \mathfrak{B}_{G}(V+W),
$$

which is the identity on objects. Precomposition with $s_{W}$ gives a functor $s_{W}^{*}: \mathcal{M}_{G}(V+W) \rightarrow \mathcal{M}_{G}(V)$. This functor has a left adjoint

$$
s_{*}^{W}: \mathcal{M}_{G}(V) \rightarrow \mathcal{M}_{G}(V+W),
$$

which is given by left Kan extension along $s_{W}$. By Lemma 3.4 of [13], the functor $s_{n}$ associated with the trivial representation $n$ is an isomorphism of categories. The functors $s_{n}^{*}$ and $s_{*}^{n}$ are therefore inverse isomorphisms of categories. We use these isomorphisms to identify $V$ - and $(V+n)$-Mackey functors, for any trivial representation $n$.

Remark 2.1. Here, and in [13], we take the objects of the category $\mathfrak{B}_{G}(V)$ to be the orbits $G / H$ of the group $G$. In [12], representations $V$ with $\left|V^{G}\right|=1$ are considered and, for that reason, the category $\mathfrak{B}_{G}(V)$ is assumed there to have a somewhat larger collection of objects. The category denoted here by $\mathfrak{B}_{G}(V)$ is denoted in [12] by $\mathfrak{B}_{G}^{0}(V)$. If $\left|V^{G}\right| \geq 2$, then, as explained in Section 5 of [12], the inclusion of $\mathfrak{B}_{G}^{0}(V)$ into the larger category induces an isomorphism between the associated categories of functors into $A b$. Thus, the difference between the definition of $\mathfrak{B}_{G}(V)$ given here and the definition given in [12] is immaterial under our assumption that $\left|V^{G}\right| \geq 2$.

If $Y$ is a based $G$-space, then the assignment of the abelian group $\left[\Sigma^{V} G / K_{+}, Y\right]_{G}$ to the orbit $G / K$ gives a $V$-Mackey functor $\underline{\pi}_{V}^{G} Y$, which is called the $V$ th homotopy functor of $Y$. The suspension maps

$$
\left[\Sigma^{V} G / K_{+}, Y\right]_{G} \stackrel{\Sigma^{W}}{\longrightarrow}\left[\Sigma^{V+W} G / K_{+}, \Sigma^{W} Y\right]_{G}
$$

for the various subgroups $K$ of $G$ fit together to form a map

$$
\sigma_{W}: \underline{\pi}_{V}^{G} Y \rightarrow s_{W}^{*} \underline{\pi}_{V+W}^{G} \Sigma^{W} Y
$$

of $V$-Mackey functors. Denote by

$$
\widetilde{\sigma}_{W}: s_{*}^{W} \underline{\pi}_{V}^{G} Y \rightarrow \underline{\pi}_{V+W}^{G} \Sigma^{W} Y
$$

the adjoint of $\sigma_{W}$. This map $\widetilde{\sigma}_{W}$ is the unstable analog of the map $\widetilde{\sigma}_{W}$ : $s_{*}^{W} \underline{\pi}_{\alpha}^{G, U} Z \rightarrow \underline{\pi}_{\alpha+W}^{G, U} \Sigma^{W} Z$ of Theorem 1.9. The unstable equivariant suspension theorem (Theorem 2.5) of [12] asserts that $\widetilde{\sigma}_{W}: s_{*}^{W} \underline{\pi}_{V}^{G} Y \rightarrow \underline{\pi}_{V+W}^{G}{ }^{\Sigma^{W}} Y$ is an isomorphism if $Y$ is appropriately connected.

The following is our extension of Theorem 2.5 of [12] to an unstable analog of Theorem 1.14.

TheOREM 2.2. Let $V$ and $W$ be finite-dimensional G-representations with $\left|V^{G}\right| \geq 2$, and let $Y$ be a based $G$-space such that $\left|V^{K}\right| \leq 2 c\left(Y^{K}\right)$, for every $K \leq G$. Then there is a right half-plane spectral sequence which 
converges in total degree $n$ to $\underline{\pi}_{V+W}^{G} \Sigma^{W-n} Y$ for every integer $n \leq 0$. In the fourth quadrant, the $E^{2}$-term of this spectral sequence is given by

$$
E_{p, q}^{2}= \begin{cases}\underline{\pi}_{V+W+p+q}^{G, U}\left(\Sigma^{W} K_{U}^{G}\left(\underline{\pi}_{V}^{G} \Sigma^{-q} Y, V+q\right)\right) & \text { for }-|V|<q \leq 0, \\ 0 & \text { for } q \leq-|V| .\end{cases}
$$

Moreover, for $q \leq 0, E_{0, q}^{2} \cong s_{*}^{W} \underline{\pi}_{V}^{G} \Sigma^{-q} Y$ and, under this isomorphism, the edge homomorphism

$$
E_{0, q}^{2} \rightarrow E_{0, q}^{\infty} \subset \underline{\pi}_{V+W}^{G} \Sigma^{W-q} Y
$$

is identified with the map

$$
\widetilde{\sigma}_{W}: s_{*}^{W} \underline{\pi}_{V}^{G} \Sigma^{-q} Y \rightarrow \underline{\pi}_{V+W}^{G} \Sigma^{W-q} Y .
$$

R e mark 2.3. In this theorem, only the fourth quadrant portion of the $E^{2}$-term of the unstable suspension spectral sequence is identified. Since the purpose of the theorem is to compute the homotopy functor $\underline{\pi}_{V+W}^{G} \Sigma^{W} Y$, to which the spectral sequence converges in total degree zero, the remainder of the $E^{2}$-term is irrelevant.

To prove Theorem 2.2, and even to ensure that the equivariant EilenbergMacLane spectra $K_{U}^{G}\left(\underline{\pi}_{V}^{G} \Sigma^{-q} Y, V+q\right)$ appearing in the statement of the theorem are well defined, we must establish isomorphisms between certain unstable homotopy functors and analogous stable homotopy functors computed in the trivial $G$-universe $U=\mathbb{R}^{\infty}$. Define the functor $s_{\infty}: \mathfrak{B}_{G}(V) \rightarrow \mathfrak{B}_{G}^{U}(V)$ to be the identity on objects and to be given on morphisms by the composite

$$
\begin{aligned}
{\left[\Sigma^{V} G / J_{+}, \Sigma^{V} G / K_{+}\right]_{G} \stackrel{\Sigma_{U}^{\infty}}{\longrightarrow}\left[\Sigma_{U}^{\infty} \Sigma^{V} G / J_{+}, \Sigma_{U}^{\infty} \Sigma^{V} G / K_{+}\right]_{G} } \\
\cong\left[S_{U}^{V} \wedge G / J_{+}, S_{U}^{V} \wedge G / K_{+}\right]_{G} .
\end{aligned}
$$

Precomposition by $s_{\infty}$ gives a functor $s_{\infty}^{*}: \mathcal{M}_{G}^{U}(V) \rightarrow \mathcal{M}_{G}(V)$. The functor $s_{\infty}^{*}$ has a left adjoint $s_{*}^{\infty}: \mathcal{M}_{G}(V) \rightarrow \mathcal{M}_{G}^{U}(V)$, given by left Kan extension along $s_{\infty}$. The stabilization maps

$$
\left[\Sigma^{V} G / K_{+}, Y\right]_{G} \stackrel{\Sigma_{U}^{\infty}}{\longrightarrow}\left[\Sigma_{U}^{\infty} \Sigma^{V} G / K_{+}, \Sigma_{U}^{\infty} Y\right]_{G} \cong\left[S_{U}^{V} \wedge G / K_{+}, \Sigma_{U}^{\infty} Y\right]_{G}
$$

for the various subgroups $K$ of $G$ fit together to form a map

$$
\sigma_{\infty}: \underline{\pi}_{V}^{G} Y \rightarrow s_{\infty}^{*} \underline{\pi}_{V}^{G, U} \Sigma_{U}^{\infty} Y
$$

of $V$-Mackey functors. The next result, which follows easily from the classical equivariant suspension theorem $[4,7,24,27]$, establishes the required isomorphisms between the stable and unstable homotopy functors.

Lemma 2.4. Let $U$ be the trivial $G$-universe and let $V$ be a $G$-representation with $\left|V^{G}\right| \geq 2$. Then

(a) The functor $s_{\infty}: \mathfrak{B}_{G}(V) \rightarrow \mathfrak{B}_{G}^{U}(V)$ is an isomorphism of categories. Thus, the functors $s_{\infty}^{*}: \mathcal{M}_{G}^{U}(V) \rightarrow \mathcal{M}_{G}(V)$ and $s_{*}^{\infty}: \mathcal{M}_{G}(V) \rightarrow \mathcal{M}_{G}^{U}(V)$ are inverse equivalences of categories. 
(b) Let $Y$ be a based $G$-space. If $\left|V^{K}\right| \leq 2 c\left(Y^{K}\right)$ for every $K \leq G$, then the map $\sigma_{\infty}: \underline{\pi}_{V}^{G} Y \rightarrow s_{\infty}^{*} \underline{\pi}_{V}^{G, U} \Sigma_{U}^{\infty} Y$ is an isomorphism.

Part (a) of this lemma and Theorem 1.2 imply that the categories $\mathfrak{B}_{G}(V)$ and $\mathfrak{B}_{G}^{U}(V+q)$ are isomorphic for every integer $q$. Thus, any $V$-Mackey functor $M$ may be regarded as a $U-(V+q)$-Mackey functor and so has an associated Eilenberg-MacLane spectrum $K_{U}^{G}(M, V+q)$ indexed on the trivial universe $U$. In particular, for every $q \leq 0$ and every $G$-space $Y$, there is an Eilenberg-MacLane spectrum $K_{U}^{G}\left(\underline{\pi}_{V}^{G} \Sigma^{-q} Y, V+q\right)$ indexed on $U$. Thus, the description of the $E^{2}$-term of our unstable spectral sequence given in Theorem 2.2 is meaningful. The remainder of this section is devoted to the proof of that theorem.

Proof of Theorem 2.2. The stable suspension spectral sequence of Theorem 1.14(a), with $U=\mathbb{R}^{\infty}, \alpha=V$, and $Z=\Sigma_{U}^{\infty} Y$, converges to $\underline{\pi}_{V+W+p+q}^{G, U} \Sigma^{W} \Sigma_{U}^{\infty} Y$. Its $E^{2}$-term is given by

$$
E_{p, q}^{2}=\underline{\pi}_{V+W+p+q}^{G, U}\left(\Sigma^{W} K_{U}^{G}\left(\underline{\pi}_{V+q}^{G, U} \Sigma_{U}^{\infty} Y, V+q\right)\right) .
$$

For any integer $q$, its edge homomorphism

$$
E_{0, q}^{2} \rightarrow E_{0, q}^{\infty} \subset \underline{\pi}_{V+W+q}^{G, U} \Sigma^{W} \Sigma_{U}^{\infty} Y
$$

may be identified with the map

$$
\widetilde{\sigma}_{W}: s_{*}^{W} \underline{\pi}_{V+q}^{G, U} \Sigma_{U}^{\infty} Y \rightarrow \underline{\pi}_{V+W+q}^{G, U} \Sigma^{W} \Sigma_{U}^{\infty} Y .
$$

Lemma 2.4(b) indicates that the maps

$$
\begin{aligned}
\sigma_{\infty}: \underline{\pi}_{V+W}^{G} \Sigma^{W-p-q} Y & \rightarrow s_{\infty}^{*} \underline{\pi}_{V+W}^{G, U} \Sigma^{\infty} \Sigma^{W-p-q} Y \\
& \cong s_{\infty}^{*} \underline{\pi}_{V+W+p+q}^{G, U} \Sigma^{\infty} \Sigma^{W} Y
\end{aligned}
$$

and

$$
\sigma_{\infty}: \underline{\pi}_{V}^{G} \Sigma^{-q} Y \rightarrow s_{\infty}^{*} \underline{\pi}_{V}^{G, U} \Sigma^{\infty} \Sigma^{-q} Y \cong s_{\infty}^{*} \underline{\pi}_{V+q}^{G, U} \Sigma_{U}^{\infty} Y
$$

are isomorphisms if $q, p+q \leq 0$. The functors $s_{\infty}^{*}$ appearing in these two isomorphisms are just the equivalences of categories that we use to identify $V$ - and $(V+W)$-Mackey functors with $U-V$ - and $U-(V+W)$-Mackey functors, respectively. Thus, the two isomorphisms $\sigma_{\infty}$ allow us to identify this special case of the stable suspension spectral sequence as a spectral sequence whose fourth quadrant $E^{2}$-term and convergence are precisely those promised by the theorem. Further, for $q \leq 0$, the isomorphisms $\sigma_{\infty}$ allow us to identify the maps $\widetilde{\sigma}_{W}: s_{*}^{W} \underline{\pi}_{V+q}^{G, U} \Sigma_{U}^{\infty} Y \rightarrow \underline{\pi}_{V+W+q}^{G, U} \Sigma^{W} \Sigma_{U}^{\infty} Y$, which are the edge homomorphisms of the stable suspension spectral sequence, with the maps

$$
\widetilde{\sigma}_{W}: s_{*}^{W} \underline{\pi}_{V}^{G} \Sigma^{-q} Y \rightarrow \underline{\pi}_{V+W}^{G} \Sigma^{W-q} Y .
$$


3. $G-C W(\alpha)$ spectra and $U$ - $\alpha$-Eilenberg-MacLane spectra. Throughout this section, $U$ is to be a $G$-universe and $\alpha$ is to be an element of $R O^{+}(G, U)$. Here, the notion of a based $G-C W(V)$-complex introduced in [12] is generalized to obtain the notion of a $G-C W(\alpha)$ spectrum indexed on $U$. This notion is used to construct $U$ - $\alpha$-Eilenberg-MacLane spectra and to show that they have the properties ascribed to them in Section 1. At the conclusion of this section, the properties of these spectra are used to prove Theorem 1.9.

Like $G-C W(V)$-complexes, $G-C W(\alpha)$ spectra are examples of the generalized $C W$ complexes introduced by May in [20,21]. The collection $\mathscr{J}$ of generalized spheres used to form $G-C W(\alpha)$ spectra is the set

$$
\left\{S_{U}^{\alpha+n} \wedge G / K_{+}\right\}_{n \geq-1, K \leq G} .
$$

One could, of course, form a type of generalized $G-C W$ spectrum using generalized spheres of the form $S_{U}^{\alpha+n} \wedge G / K_{+}$where $n$ was allowed to be an arbitrary integer. However, our primary interest in $G-C W(\alpha)$ spectra is that they provide models of spectra that are at least as connected as the spectrum $S_{U}^{\alpha}$. In order to obtain spectra with this sort of connectivity, we insist that $n \geq-1$. Almost all of the results in this section, and their proofs, are direct transcriptions of results about based $G-C W(V)$-complexes and $G-V$ Eilenberg-MacLane spaces found in Sections 3 and 4 of [12]. Thus, here, we merely record the translations of the most important items from those two sections of [12] and mention the few instances in which the arguments given there must be adjusted in order to obtain the analogous results for spectra.

A $G-C W(\alpha)$ spectrum indexed on $U$ is, of course, just a spectrum $Y$ indexed on $U$ together with a filtration $\left\{Y^{k}\right\}_{k>-1}$ of $Y$ by closed subspectra such that $Y=\operatorname{colim}_{k} Y^{k}, Y^{-1}=*$, and, for $k \geq-1, Y^{k+1}$ is the cofibre of a map $\lambda_{k}: \bigvee_{j \in J_{k}} S_{U}^{\alpha+k} \wedge\left(G / K_{j}\right)_{+} \rightarrow Y^{k}$. Cells, skeleta, subcomplexes, relative $G-C W(\alpha)$ spectra, degree, cellular maps, etc., are defined just as in [12]. It is easy to see that a $G-C W(\alpha)$ spectrum has the $G$-homotopy type of a $G-C W$ spectrum. Similarly, Lemma 3.4 of [12], which deals with $C W$ structures on wedges and cylinders, obviously generalizes to $G-C W(\alpha)$ spectra.

The connectivity of $G$-spectra, and of maps between $G$-spectra, is, of course, usually measured with the homotopy functors $\underline{\pi}_{n}^{G, U}$, for $n \in \mathbb{Z}$. It is expressed, like the connectivity of $G$-spaces and maps, in terms of dimension functions $[12,13]$. However, in the context of spectra, $-\infty$ and negative integers less than -1 are meaningful values for dimension functions. If the element $\beta$ in $R O(G)$ is represented by the formal difference $V-W$, then the associated dimension function $\left|\beta^{*}\right|$ takes the value $\left|\beta^{K}\right|=\left|V^{K}\right|-\left|W^{K}\right|$ on a subgroup $K$ of $G$. In the context of $G-C W(\alpha)$ spectra, it is sometimes more natural to measure the connectivity of spectra, and of maps between spectra, in terms of the homotopy functors $\underline{\pi}_{\alpha+n}^{G, U}$. In particular, there is a 
spectrum-level generalization of the notion of a $V$-equivalence of order $n$ introduced in [12].

Definition 3.1. Let $e: Y \rightarrow Z$ be a map between $G$-spectra indexed on $U$, and let $n \geq 0$. The map $e$ is an $\alpha$-equivalence of order $n$ if the map

$$
e_{*}: \underline{\pi}_{\alpha+k}^{G, U} Y \rightarrow \underline{\pi}_{\alpha+k}^{G, U} Z
$$

is a monomorphism for $-1 \leq k \leq n-1$ and an epimorphism for $0 \leq k \leq n$. The map $e$ is a weak $\alpha$-equivalence if the map $e_{*}$ is an $\alpha$-equivalence of order $n$ for all $n \geq 0$.

With the notions of an $\alpha$-equivalence of order $n$ and a weak $\alpha$-equivalence replacing the corresponding $V$-notions and with the dimension function $\left|\alpha^{*}\right|$ replacing the dimension function $\left|V^{*}\right|$, the results in [12], like Lemmas 3.3 and 3.7 and Theorem 3.8 (HELP), concerning the connectivity of $G-C W(V)$ spaces, and of maps between spaces, generalize to results about the connectivity of $G-C W(\alpha)$ spectra and of maps between spectra. Proposition 2.4(i) of [13] provides the one technical result about the connectivity of spectra that is needed to prove all of these results. The HELP property of $G-C W(\alpha)$ spectra formally implies a cellular approximation theorem for maps between $G-C W(\alpha)$ spectra. It also implies the following Whitehead theorem for $G-C W(\alpha)$ spectra.

Theorem 3.2. Let $n \geq 0, X$ be a $G$-CW $(\alpha)$ spectrum, and $e: Y \rightarrow Z$ be an $\alpha$-equivalence of order $n$ between two $G$-spectra indexed on $U$. Then $e_{*}:[X, Y]_{G} \rightarrow[X, Z]_{G}$ is an isomorphism if the degree of $X$ is less than $n$ and an epimorphism if the degree of $X$ is $n$. Moreover, if $e$ is a weak $\alpha$-equivalence, then $e_{*}$ is an isomorphism for every $G-C W(\alpha)$ spectrum $X$. Thus, if $Y$ and $Z$ are $G$-CW $(\alpha)$ spectra and e is a weak $\alpha$-equivalence, then $e$ is a G-homotopy equivalence.

Lemma 3.5 of [12], which describes the behavior of $V$-Mackey functors of the form $\underline{\pi}_{V}^{G}\left(\bigvee_{j \in J} \Sigma^{V}\left(G / K_{j}\right)_{+}\right)$, generalizes easily to a result describing the behavior of $U$ - $\alpha$-Mackey functors of the form $\underline{\pi}_{\alpha}^{G, U}\left(\bigvee_{j \in J} S_{U}^{\alpha} \wedge\left(G / K_{j}\right)_{+}\right)$. The proof of Lemma 3.5(a) of [12] requires the observation that a map of a compact space into an infinite wedge of spaces factors through a finite subwedge. The proof of Proposition 4.2 in the Appendix of [18] provides the appropriate spectrum-level analog of this assertion.

For our purposes, the most significant property of $G-C W(\alpha)$ spectra is that they provide good models for spectra that are $(\alpha-1)$-connected, just as based $G-C W(V)$-complexes provide good models for $G$-spaces that are $\left(\left|V^{*}\right|-1\right)$-connected. Recall, from Definition 1.8 , that $\widetilde{h} G \mathcal{S}(U, \alpha)$ is the full subcategory of the $U$-stable category $\widetilde{h} G \mathcal{S} U$ whose objects are the $(\alpha-1)$-connected spectra. The proof of the following approximation result 
is essentially identical to the proof of Proposition 3.11 in [12].

Proposition 3.3. The inclusion functor $\iota: \widetilde{h} G \mathcal{S}(U, \alpha) \rightarrow \widetilde{h} G \mathcal{S} U$ has a right adjoint

$$
\Gamma_{\alpha}: \widetilde{h} G \mathcal{S} U \rightarrow \widetilde{h} G \mathcal{S}(U, \alpha)
$$

For any $G$-spectrum $Y$ indexed on $U, \Gamma_{\alpha} Y$ is a $G$-CW $(\alpha)$ spectrum, and the counit $\varepsilon_{\alpha}: \iota \Gamma_{\alpha} Y \rightarrow Y$ of the adjunction is a weak $\alpha$-equivalence. Thus, if $Y$ is in $\widetilde{h} G \mathcal{S}(U, \alpha)$, then $\varepsilon_{\alpha}$ is an isomorphism in $\widetilde{h} G \mathcal{S} U$.

Remark 3.4. Let $W$ be a finite-dimensional $G$-representation. The functor $\Sigma^{W}$ obviously converts $G$ - $C W(\alpha)$ spectra into $G-C W(\alpha+W)$ spectra. This fact, and the observation that any $(\alpha-1)$-connected spectrum is weakly equivalent to a $G-C W(\alpha)$ spectrum, imply that the functor $\Sigma^{W}$ converts $(\alpha-1)$-connected spectra into $(\alpha+W-1)$-connected spectra. Similarly, if $\iota: U \rightarrow U^{\prime}$ is a linear isometry between the $G$-universes $U$ and $U^{\prime}$, then the functor $\iota_{*}$ takes $G-C W(\alpha)$ spectra indexed on $U$ to $G$ - $C W(\alpha)$ spectra indexed on $U^{\prime}$. It therefore also takes $(\alpha-1)$-connected spectra indexed on $U$ to $(\alpha-1)$-connected spectra indexed on $U^{\prime}$.

We often work with the sequence of approximations $\varepsilon_{\alpha+q}: \Gamma_{\alpha+q} Y \rightarrow Y$, for $q \in \mathbb{Z}$. In this context, $\Gamma_{\alpha+q} Y$ may be regarded as the $(\alpha+q-1)$ connected cover of $Y$ in the sense that it looks identical to $Y$ in the eyes of the functors $\underline{\pi}_{\alpha+n}^{G, U}$, for $n \geq q$, but vanishes in the eyes of the functors $\pi_{\alpha+n}^{G, U}$, for $n<q$. Thus, for $q \in \mathbb{Z}$, we denote the spectrum $\Gamma_{\alpha+q} Y$ by $Y_{\alpha}[q, \infty)$ and the map $\varepsilon_{\alpha+q}$ by $\varepsilon_{q}^{\alpha}: Y_{\alpha}[q, \infty) \rightarrow Y$.

The connectivity properties of $G-C W(\alpha)$ spectra make them an ideal tool for constructing $U$ - $\alpha$-Eilenberg-MacLane spectra. The following generalization of Proposition 4.1 and Lemma 4.2 of [12] is proved in the same fashion as those results and supplies all that is needed to complete the proof of Theorem 1.13.

Proposition 3.5. Let $M$ be a $U$ - $\alpha$-Mackey functor. Then there is a $G-C W(\alpha)$ spectrum $K_{U}^{G}(M, \alpha)$ such that $\underline{\pi}_{\alpha}^{G, U} K_{U}^{G}(M, \alpha)=M$ and, for every nonzero integer $k, \underline{\pi}_{\alpha+k}^{G, U} K_{U}^{G}(M, \alpha)=0$. Moreover, if $Y$ is an $(\alpha-1)$ connected spectrum, then the map

$$
\pi:\left[Y, K_{U}^{G}(M, \alpha)\right] \rightarrow \mathcal{M}_{G}^{U}(\alpha)\left(\underline{\pi}_{\alpha}^{G, U} Y, \underline{\pi}_{\alpha}^{G, U} K_{U}^{G}(M, \alpha)\right),
$$

induced by the functor $\underline{\pi}_{\alpha}^{G, U}$, is an isomorphism.

The proof of the space-level equivariant suspension theorem in [12] makes use of three basic components. These are the adjunction relating EilenbergMacLane spaces and homotopy groups given in Theorem 1.5 of [12], the $V$-Mackey functor formulation of the $\left(\Sigma^{W}, \Omega^{W}\right)$-adjunction given in Lemma 1.6(a) of [12], and the natural isomorphism $\theta$ of Lemma 1.6(b) of [12] describing the effect of the loop functor $\Omega^{W}$ on Eilenberg-MacLane spaces. The 
proof of Theorem 1.9, which is a spectrum-level variant of the space-level suspension theorem of [12], uses three similar components. Theorem 1.13 provides the adjunction relating Eilenberg-MacLane spectra and stable homotopy groups. The following two lemmas, whose proofs are identical to those of the corresponding results in [12], provide the other two components.

Lemma 3.6. (a) Let $W$ be a finite-dimensional $G$-representation. For every $G$-spectrum $Z$ indexed on $U$, there is an isomorphism

$$
\phi_{W}: \underline{\pi}_{\alpha}^{G, U} \Omega^{W} Z \rightarrow s_{W}^{*} \underline{\pi}_{\alpha+W}^{G, U} Z
$$

of $U$ - $\alpha$-Mackey functors. This isomorphism is natural in $Z$.

(b) Let $\iota: U \rightarrow U^{\prime}$ be a linear $G$-isometry from $U$ into another $G$-universe $U^{\prime}$. For every $G$-spectrum $Z^{\prime}$ indexed on $U^{\prime}$, there is an isomorphism

$$
\phi_{\iota}: \underline{\pi}_{\alpha}^{G, U} \iota^{*} Z^{\prime} \rightarrow s_{\iota}^{*} \underline{\pi}_{\alpha}^{G, U^{\prime}} Z^{\prime}
$$

of $U$ - $\alpha$-Mackey functors. This isomorphism is natural in $Z^{\prime}$.

Lemma 3.7. (a) For each finite-dimensional $G$-representation $W$ and each $U-(\alpha+W)$-Mackey functor $N$, there is a $G$-homotopy equivalence

$$
\theta_{W}: \Omega^{W} K_{U}^{G}(N, \alpha+W) \rightarrow K_{U}^{G}\left(s_{W}^{*} N, \alpha\right)
$$

making the diagram

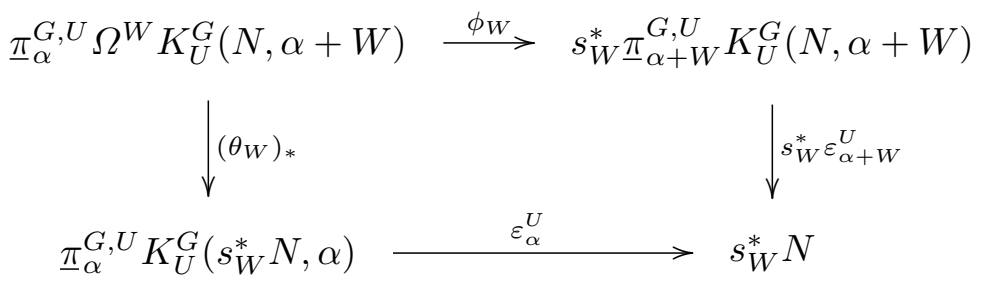

commute. This homotopy equivalence is natural in $N$.

(b) If $\iota: U \rightarrow U^{\prime}$ is a linear $G$-isometry from $U$ into another $G$-universe $U^{\prime}$ and $N$ is a $U^{\prime}-\alpha$-Mackey functor, then there is a G-homotopy equivalence

$$
\theta_{\iota}: \iota^{*} K_{U^{\prime}}^{G}(N, \alpha) \rightarrow K_{U}^{G}\left(s_{\iota}^{*} N, \alpha\right)
$$

making the diagram

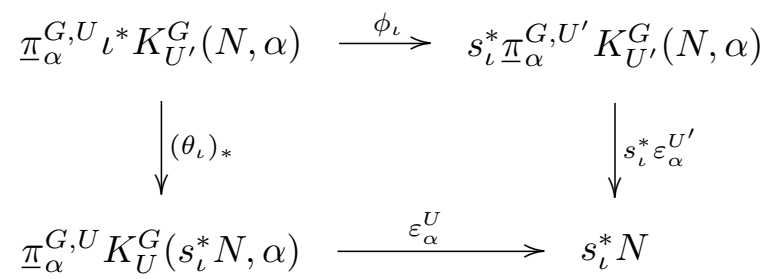

commute. This homotopy equivalence is natural in $N$. 
The first isomorphism of Theorem 1.9 follows, by an argument like that given for Theorem 2.5 of [12], from Theorem 1.13, Lemma 3.6(a), Lemma $3.7(\mathrm{a})$, and the observation that the left adjoints of the functors $K_{U}^{G}\left(s_{W}^{*} ?, \alpha\right)$ and $\Omega^{W} K_{U}^{G}(?, \alpha+W)$ are $s_{*}^{W} \underline{\pi}_{\alpha}^{G, U}$ and $\underline{\pi}_{\alpha+W}^{G, U} \Sigma^{W}$, respectively. The second isomorphism of Theorem 1.9 follows in the same way from the second parts of the two lemmas and the observation that the left adjoints of the functors $K_{U}^{G}\left(s_{\iota}^{*} ?, \alpha\right)$ and $\iota^{*} K_{U^{\prime}}^{G}(?, \alpha)$ are $s_{*}^{\iota} \underline{\pi}_{\alpha}^{G, U}$ and $\underline{\pi}_{\alpha}^{G, U^{\prime}} \iota_{*}$, respectively.

4. $\alpha$-Postnikov towers and the proof of Theorem 1.14. Throughout this section, $U$ is to be a $G$-universe and $\alpha$ is to be an element of $R O^{+}(G, U)$. Here we construct, for each $G$-spectrum $Z$ indexed on $U$, a generalized Postnikov tower $\left\{Z_{\alpha}(-\infty, q]\right\}_{q \in \mathbb{Z}}$ in which the homotopy groups $\underline{\pi}_{\alpha+q}^{G, U} Z, q \in \mathbb{Z}$, are added sequentially in the same way that the homotopy groups $\underline{\pi}_{q}^{G, U} Z$ are added sequentially in an ordinary Postnikov tower. We then show that the two spectral sequences of Theorem 1.14 can be obtained from the filtrations $\left\{\Sigma^{W} Z_{\alpha}(-\infty, q]\right\}$ of $\Sigma^{W} Z$ and $\left\{\iota_{*} Z_{\alpha}(-\infty, q]\right\}$ of $\iota_{*} Z$.

The $\alpha$-Postnikov tower of a $G$-spectrum $Z$ is constructed from the $(\alpha+q)$ connected covers $Z_{\alpha}[q+1, \infty)$ of $Z$ in the same way that the ordinary Postnikov tower of a nonequivariant spectrum $X$ is constructed from its $q$-connected covers $X[q+1, \infty)$ (see, for example, $[2,19]$ ).

Construction 4.1. (a) Let $Z$ be a $G$-spectrum indexed on $U$. For each integer $q$, let $Z_{\alpha}(-\infty, q]$ be the cofibre of the map $\varepsilon_{q+1}^{\alpha}: Z_{\alpha}[q+1, \infty) \rightarrow Z$ defined in Section 3. Clearly, for $n \leq q$, the functor $\pi_{\alpha+n}^{G, U}$ converts the projection map $\pi_{q}: Z \rightarrow Z_{\alpha}(-\infty, q]$ into an isomorphism. However, for $n>q$, $\underline{\pi}_{\alpha+n}^{G, U} Z_{\alpha}(-\infty, q]=0$. It is therefore reasonable to think of $Z_{\alpha}(-\infty, q]$ as the $q$ th stage in a generalized Postnikov tower for $Z$ which is intended to add the homotopy functors $\underline{\pi}_{\alpha+q}^{G, U} Z$ sequentially to $Z$. To complete the construction of this tower, we must produce a suitable map $i_{q}$ from $Z_{\alpha}(-\infty, q]$ to $Z_{\alpha}(-\infty, q-1]$ for each integer $q$. Since the map $\varepsilon_{q}^{\alpha}: Z_{\alpha}[q, \infty) \rightarrow Z$ is a weak $(\alpha+q)$-equivalence and $Z_{\alpha}[q+1, \infty)$ is $(\alpha+q)$-connected, the map

$$
\left(\varepsilon_{q}^{\alpha}\right)_{*}:\left[Z_{\alpha}[q+1, \infty), Z_{\alpha}[q, \infty)\right] \rightarrow\left[Z_{\alpha}[q+1, \infty), Z\right]
$$

is an isomorphism by Theorem 3.2. It follows that there is a map

$$
\gamma_{q+1}: Z_{\alpha}[q+1, \infty) \rightarrow Z_{\alpha}[q, \infty)
$$

such that $\varepsilon_{q+1}^{\alpha} \simeq \varepsilon_{q}^{\alpha} \circ \gamma_{q+1}$. The weak universal property of cofibres implies the existence of a map

$$
i_{q}: Z_{\alpha}(-\infty, q] \rightarrow Z_{\alpha}(-\infty, q-1]
$$


making the diagram

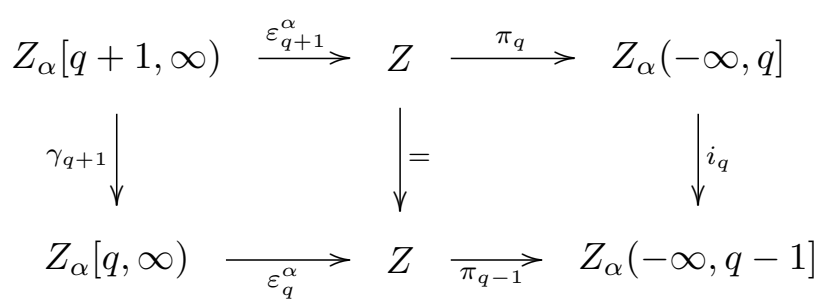

of cofibre sequences commute in $\widetilde{h} G \mathcal{S} U$. In fact, since the homotopy set $\left[\Sigma Z_{\alpha}[q+1, \infty), Z_{\alpha}(-\infty, q-1]\right]_{G}$ vanishes, there is a unique map $i_{q}$ in $h G \mathcal{S} U$ making the right square commute. From the diagram, it follows that the map

$$
\left(i_{q}\right)_{*}: \underline{\pi}_{\alpha+n}^{G, U} Z_{\alpha}(-\infty, q] \rightarrow \underline{\pi}_{\alpha+n}^{G, U} Z_{\alpha}(-\infty, q-1]
$$

is an isomorphism for $n<q$. Moreover, the fibre of the map $i_{q}$ has the weak homotopy type of the Eilenberg-MacLane spectrum $K_{U}^{G}\left(\underline{\pi}_{\alpha+q}^{G, U} Z, \alpha+q\right)$. We refer to the $\mathbb{Z}$-indexed collection of spectra $\left\{Z_{\alpha}(-\infty, q]\right\}$, together with the collections $\left\{\pi_{q}: Z \rightarrow Z_{\alpha}(-\infty, q]\right\}$ and $\left\{i_{q}: Z_{\alpha}(-\infty, q] \rightarrow Z_{\alpha}(-\infty, q-1]\right\}$ of maps, as the $\alpha$-Postnikov tower of $Z$.

(b) In order to show that the spectral sequences of Theorem 1.14 are natural in $Z$, we must show that our Postnikov tower construction is natural. Let $f: Y \rightarrow Z$ be a map of $G$-spectra indexed on $U$. Theorem 3.2 indicates that, for each integer $q$, there is a map $f_{\alpha}[q+1, \infty): Y_{\alpha}[q+1, \infty) \rightarrow Z_{\alpha}[q+1, \infty)$ making the left square of the diagram

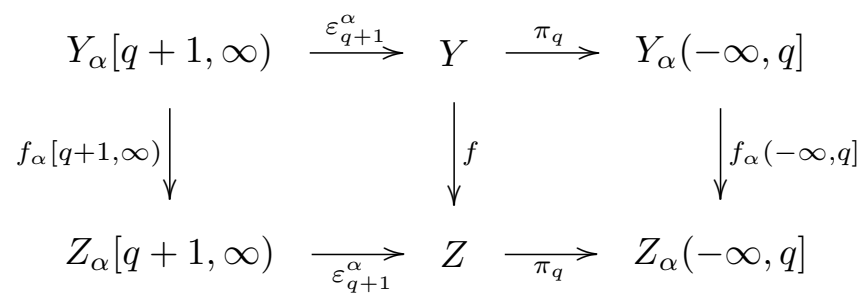

of cofibre sequences commute in $\widetilde{h} G \mathcal{S} U$. The weak universality of cofibres implies the existence of a map $f_{\alpha}(-\infty, q]$ making the right square commute in $\widetilde{h} G \mathcal{S} U$. In fact, by an argument like that used for the map $i_{q}$, there is only one map $f_{\alpha}(-\infty, q]$ in $\widetilde{h} G \mathcal{S} U$ which makes the right square of the diagram above commute in $\widetilde{h} G \mathcal{S} U$. To prove that the collection of maps

$$
\left\{f_{\alpha}(-\infty, q]: Y_{\alpha}(-\infty, q] \rightarrow Z_{\alpha}(-\infty, q]\right\}
$$

forms a map from the $\alpha$-Postnikov tower of $Y$ to the $\alpha$-Postnikov tower of $Z$, we must show that, for each integer $q$, the pair of maps $i_{q} \circ f_{\alpha}(-\infty, q]$ and $f_{\alpha}(-\infty, q-1] \circ i_{q}$ from $Y_{\alpha}(-\infty, q]$ to $Z_{\alpha}(-\infty, q-1]$ are equal. It is 
easy to see that these two maps become equal when composed with the map $\pi_{q}: Y \rightarrow Y_{\alpha}(-\infty, q]$. This fact and the vanishing of the homotopy set $\left[\Sigma Y_{\alpha}[q+1, \infty), Z_{\alpha}(-\infty, q-1]\right]_{G}$ imply that the initial pair of maps are equal. Our $\alpha$-Postnikov tower construction is therefore functorial in $\widetilde{h} G \mathcal{S} U$.

Since the sequence of maps $\left\{i_{q}: Z_{\alpha}(-\infty, q] \rightarrow Z_{\alpha}(-\infty, q-1]\right\}$ is $\mathbb{Z}$-graded, it has both a telescope $\operatorname{Tel} Z_{\alpha}(-\infty, q]$ and a microscope Mic $Z_{\alpha}(-\infty, q]$. The following result indicates that these two constructions behave as they ought to.

Lemma 4.2. (a) Let $\pi: Z \rightarrow$ Mic $Z_{\alpha}(-\infty, q]$ be the map induced by the maps $\pi_{q}: Z \rightarrow Z_{\alpha}(-\infty, q]$. Then $\pi$ is a weak equivalence.

(b) The spectrum $\mathrm{Tel} Z_{\alpha}(-\infty, q]$ has the weak homotopy type of the onepoint spectrum *.

Proof. The map $\pi: Z \rightarrow$ Mic $Z_{\alpha}(-\infty, q]$ is easily seen to be a weak $(\alpha+q)$-equivalence for every integer $q$. It is therefore a weak equivalence. For each integer $n, \underline{\pi}_{\alpha+n}^{G, U} \operatorname{Tel} Z_{\alpha}(-\infty, q]=0$ since $\underline{\pi}_{\alpha+n}^{G, U} Z_{\alpha}(-\infty, q]$ vanishes for $q<n$. It follows that the projection $\operatorname{Tel} Z_{\alpha}(-\infty, q] \rightarrow *$ is a weak equivalence.

In order to complete the proof of Theorem 1.14, it suffices to study the effect of applying the functors $\Sigma^{W}$ and $\iota_{*}$ to the $\alpha$-Postnikov tower of the $G$-spectrum $Z$.

Proof of Theorem 1.14. We prove part (b); the proof of part (a) is essentially the same. Let $\iota: U \rightarrow U^{\prime}$ be a linear isometry between the $G$-universes $U$ and $U^{\prime}$, and let $Z$ be a $G$-spectrum indexed on $U$. For each integer $q$, let $\widetilde{Z}_{q}$ be the fibre of the map $i_{q}: Z_{\alpha}(-\infty, q] \rightarrow Z_{\alpha}(-\infty, q-1]$ so that $\widetilde{Z}_{q}$ is isomorphic to $K_{U}^{G}\left(\underline{\pi}_{\alpha+q}^{G, U} Z, \alpha+q\right)$ in $\widetilde{h} G \mathcal{S} U$. Since the functor $\iota_{*}: G \mathcal{S U} \rightarrow G \mathcal{S} U^{\prime}$ preserves fibre sequences, the sequence

$$
\iota_{*} \widetilde{Z}_{q} \rightarrow \iota_{*} Z_{\alpha}(-\infty, q] \rightarrow \iota_{*} Z_{\alpha}(-\infty, q-1]
$$

is a fibre sequence in $\widetilde{h} G \mathcal{S} U^{\prime}$. Taking

$$
D_{p, q}^{2}=\underline{\pi}_{\alpha+p+q+1}^{G, U_{*}^{\prime}} Z_{\alpha}(-\infty, q]
$$

and

$$
E_{p, q}^{2}=\underline{\pi}_{\alpha+p+q^{\iota *}}^{G, U^{\prime}} \widetilde{Z}_{q},
$$

we obtain an exact couple with maps $i: D^{2} \rightarrow D^{2}$ of bidegree $(1,-1)$, $j: D^{2} \rightarrow E^{2}$ of bidegree $(-1,1)$, and $k: E^{2} \rightarrow D^{2}$ of bidegree $(-1,0)$. These bidegrees are not the usual bidegrees for a homology exact couple. Nevertheless, since the bidegree of the composite $d=j \circ k$ is $(-2,1)$, this exact couple yields a homology spectral sequence whose $E^{2}$-term has precisely the form asserted in Theorem 1.14(b). The functoriality of our $\alpha$-Postnikov 
tower construction implies that this exact couple, and therefore its associated spectral sequence, is functorial in $Z$.

In order to show that this spectral sequence converges in total degree $n$ to $\underline{\pi}_{\alpha+n}^{G, U^{\prime}} \iota_{*} Z$, we have to produce an appropriately behaved filtration $\left\{F_{p} \underline{\pi}_{\alpha+n}^{G, U^{\prime}} \iota_{*} Z\right\}$ of $\underline{\pi}_{\alpha+n}^{G, U^{\prime}} \iota_{*} Z$ and show that

$$
E_{p, q}^{\infty} \cong F_{p} \underline{\pi}_{\alpha+p+q}^{G, U^{\prime}} Z / F_{p-1} \underline{\pi}_{\alpha+p+q}^{G, U^{\prime}} \iota_{*} Z .
$$

Define the filtration $\left\{F_{p} \underline{\pi}_{\alpha+n}^{G, U^{\prime}} \iota_{*} Z\right\}$ by

$$
F_{p} \underline{\pi}_{\alpha+n}^{G, U^{\prime}} \iota_{*} Z=\operatorname{ker}\left(\left(\iota_{*} \pi_{n-p-1}\right)_{*}: \underline{\pi}_{\alpha+n}^{G, U^{\prime}} \iota_{*} Z \rightarrow \underline{\pi}_{\alpha+n}^{G, U^{\prime}} \iota_{*} Z_{\alpha}(-\infty, n-p-1]\right) .
$$

To see that this filtration is bounded below, observe that the fibre $F \pi_{n-p-1}$ of the map

$$
\pi_{n-p-1}: Z \rightarrow Z_{\alpha}(-\infty, n-p-1]
$$

is $(\alpha+n-p-1)$-connected. The fibre $F \iota_{*} \pi_{n-p-1}$ must also be $(\alpha+n-p-1)$ connected since it is isomorphic in $\widetilde{h} G \mathcal{S} U^{\prime}$ to $\iota_{*} F \pi_{n-p-1}$. It follows that $F_{p} \underline{\pi}_{\alpha+n}^{G, U^{\prime}} \iota_{*} Z=0$ if $p<0$.

To see that $\underline{\pi}_{\alpha+n}^{G, U^{\prime}} \iota_{*} Z=\bigcup_{p} F_{p} \underline{\pi}_{\alpha+n}^{G, U^{\prime}} \iota_{*} Z$, observe that

$$
\operatorname{colim} \underline{\pi}_{\alpha+n}^{G, U^{\prime}} \iota_{*} Z_{\alpha}(-\infty, n-p-1] \cong \underline{\pi}_{\alpha+n}^{G, U^{\prime}} \operatorname{Tel} \iota_{*} Z_{\alpha}(-\infty, n-p-1],
$$

where the colimit and the telescope are taken over the maps

$$
\iota_{*} i_{n-p-1}: \iota_{*} Z_{\alpha}(-\infty, n-p-1] \rightarrow \iota_{*} Z_{\alpha}(-\infty, n-p-2] .
$$

The functor $\iota_{*}$ preserves telescopes, so Tel $\iota_{*} Z_{\alpha}(-\infty, n-p-1] \cong * \operatorname{in} \widetilde{h} G \mathcal{S} U^{\prime}$. Thus, $\operatorname{colim} \underline{\pi}_{\alpha+n}^{G, U^{\prime}} \iota_{*} Z_{\alpha}(-\infty, n-p-1]=0$. It follows by the exactness of sequential colimits that $\underline{\pi}_{\alpha+n}^{G, U^{\prime}} \iota_{*} Z=\bigcup_{p} F_{p} \underline{\pi}_{\alpha+n}^{G, U^{\prime}} \iota_{*} Z$.

To see that our filtration is properly related to the $E^{\infty}$-term of our spectral sequence, recall from [22] that $E_{p, q}^{\infty} \cong Z_{p, q}^{\infty} / B_{p, q}^{\infty}$, where

$$
Z_{p, q}^{\infty}=\bigcap_{r=2}^{\infty} k^{-1}\left(\operatorname{im} i^{r-1}: D_{p-r, q+r-1}^{2} \rightarrow D_{p-1, q}^{2}\right)
$$

and

$$
B_{p, q}^{\infty}=\bigcup_{r=2}^{\infty} j\left(\operatorname{ker} i^{r-1}: D_{p+1, q-1}^{2} \rightarrow D_{p+r, q-r}^{2}\right) .
$$

The map

$$
\left(\iota_{*} \pi_{q+r-1}\right)_{*}: \underline{\pi}_{\alpha+p+q}^{G, U^{\prime}} \iota_{*} Z \rightarrow \underline{\pi}_{\alpha+p+q}^{G, U^{\prime}} \iota_{*} Z_{\alpha}(-\infty, q+r-1]=D_{p-r, q+r-1}^{2}
$$

is surjective if $r \geq p$ since the cofibre of $\pi_{q+r-1}: Z \rightarrow Z_{\alpha}(-\infty, q+r-1]$, and therefore also of $\iota_{*} \pi_{q+r-1}$, is $(\alpha+q+r)$-connected. Thus,

$$
Z_{p, q}^{\infty}=k^{-1}\left(\operatorname{im}\left(\iota_{*} \pi_{q}\right)_{*}: \underline{\pi}_{\alpha+p+q}^{G, U^{\prime}} \iota_{*} Z \rightarrow \underline{\pi}_{\alpha+p+q}^{G, U^{\prime}} \iota_{*} Z_{\alpha}(-\infty, q]=D_{p-1, q}^{2}\right) .
$$


Moreover,

$$
D_{p+1, q-1}^{2}=\bigcup_{r=2}^{\infty}\left(\operatorname{ker} i^{r-1}: D_{p+1, q-1}^{2} \rightarrow D_{p+r, q-r}^{2}\right)
$$

by an argument like that used to show that our filtration $\left\{F_{p} \underline{\pi}_{\alpha+n}^{G, U^{\prime}} \iota_{*} Z\right\}$ converges to $\underline{\pi}_{\alpha+n}^{G, U^{\prime}} \iota_{*} Z$. It follows that

$$
B_{p, q}^{\infty}=j\left(D_{p+1, q-1}^{2}\right)=\operatorname{ker}\left(k: E_{p, q}^{2} \rightarrow D_{p-1, q}^{2}\right) .
$$

The map $k: E_{p, q}^{2} \rightarrow D_{p-1, q}^{2}$ therefore induces an isomorphism

$$
E_{p, q}^{\infty} \cong\left(\operatorname{im} k: E_{p, q}^{2} \rightarrow D_{p-1, q}^{2}\right) \cap\left(\operatorname{im}\left(\iota_{*} \pi_{q}\right)_{*}: \underline{\pi}_{\alpha+p+q}^{G, U^{\prime}} \iota_{*} Z \rightarrow D_{p-1, q}^{2}\right) .
$$

Since

$$
\begin{aligned}
\operatorname{im}(k & \left.: E_{p, q}^{2} \rightarrow D_{p-1, q}^{2}\right)=\operatorname{ker}\left(i: D_{p-1, q}^{2} \rightarrow D_{p, q-1}^{2}\right) \\
& =\operatorname{ker}\left(\left(\iota_{*} i_{q}\right)_{*}: \underline{\pi}_{\alpha+p+q}^{G, U^{\prime}} \iota_{*} Z_{\alpha}(-\infty, q] \rightarrow \underline{\pi}_{\alpha+p+q_{*}}^{G, U^{\prime}} Z_{\alpha}(-\infty, q-1]\right),
\end{aligned}
$$

the map

$$
\left(\iota_{*} \pi_{q}\right)_{*}: \underline{\pi}_{\alpha+p+q^{\iota *}}^{G, U^{\prime}} Z \rightarrow \underline{\pi}_{\alpha+p+q^{\prime}}^{G, U^{\prime}} Z_{\alpha}(-\infty, q]=D_{p-1, q}^{2}
$$

induces the desired isomorphism

$$
F_{p} \underline{\pi}_{\alpha+p+q}^{G, U^{\prime}} \iota_{*} Z / F_{p-1} \underline{\pi}_{\alpha+p+q}^{G, U^{\prime}} \iota_{*} Z \cong E_{p, q}^{\infty} .
$$

All that is still lacking in the proof of Theorem 1.14(b) is a verification of the assertion in the theorem about the edge homomorphisms in our spectral sequence. Let $F_{q}$ be the fibre of the map $\pi_{q}: Z \rightarrow Z_{\alpha}(-\infty, q]$. Observe that $F_{q}$ is $(\alpha+q)$-connected. The weak universality of fibres provides a map $\delta: F_{q-1} \rightarrow \widetilde{Z}_{q}$ making the diagram

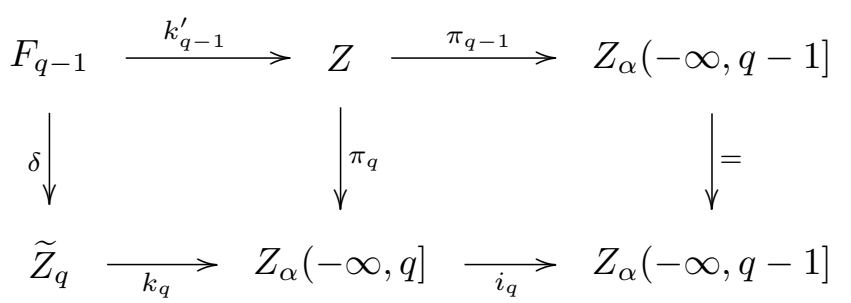

of fibre sequences commute. Observe that the functor $\underline{\pi}_{\alpha+q}^{G, U}$ converts the four maps in the left square of this diagram into isomorphisms. We use these isomorphisms to identify the homotopy functors $\underline{\pi}_{\alpha+q}^{G, U} F_{q-1}$ and $\underline{\pi}_{\alpha+q}^{G, U} \widetilde{Z}_{q}$ with $\underline{\pi}_{\alpha+q}^{G, U} Z$. Applying the functor $\underline{\pi}_{\alpha+q}^{G, U^{\prime}} \iota_{*}$ to this diagram and identifying the appropriate entries in terms of our exact couple, we obtain the commutative 
diagram

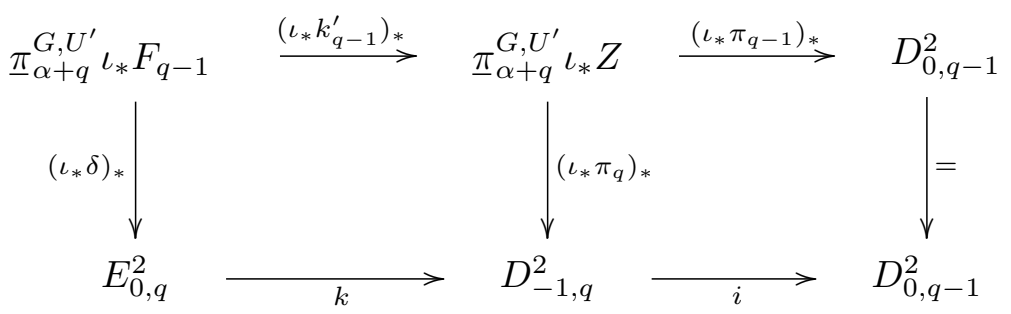

It follows from the connectivity of $F_{q}$ that the vertical arrows $\left(\iota_{*} \delta\right)_{*}$ and $\left(\iota_{*} \pi_{q}\right)_{*}$ in this diagram are isomorphisms. Our earlier discussion of the convergence of our spectral sequence allows us to identify $E_{0, q}^{\infty}$ with the image of the map $k: E_{0, q}^{2} \rightarrow D_{-1, q}^{2}$ in this diagram. Under this identification, the composite

$$
E_{0, q}^{2} \rightarrow E_{0,2}^{\infty} \cong F_{0} \underline{\pi}_{\alpha+q}^{G, U^{\prime}} \iota_{*} Z \subset \underline{\pi}_{\alpha+q}^{G, U^{\prime}} \iota_{*} Z
$$

may be identified with the composite $\left(\iota_{*} k_{q-1}^{\prime}\right)_{*} \circ\left(\left(\iota_{*} \delta\right)_{*}\right)^{-1}$. By Theorem 1.9 , the maps

$$
\begin{aligned}
& \widetilde{\sigma}_{\iota}: s_{*}^{\iota} \underline{\pi}_{\alpha+q}^{G, U} \widetilde{Z}_{q} \rightarrow \underline{\pi}_{\alpha+q}^{G, U^{\prime}} \iota_{*} \widetilde{Z}_{q}=E_{0, q}^{2} \quad \text { and } \\
& \widetilde{\sigma}_{\iota}: s_{*}^{\iota} \underline{\pi}_{\alpha+q}^{G, U} F_{q-1} \rightarrow \underline{\pi}_{\alpha+q}^{G, U^{\prime}} \iota_{*} F_{q-1}
\end{aligned}
$$

are isomorphisms. The commutative diagram

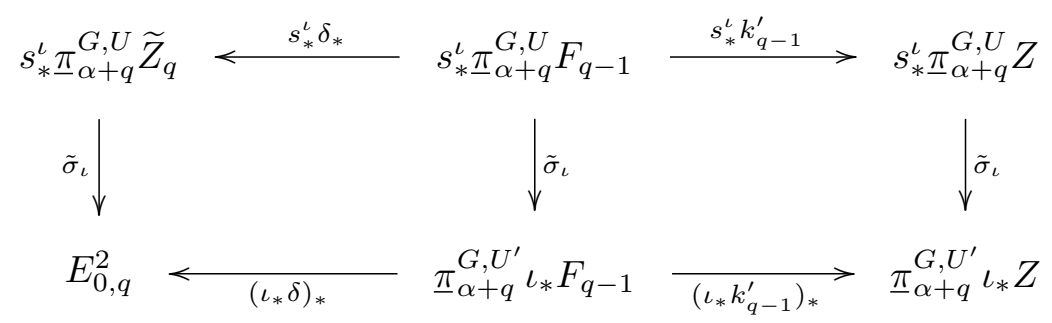

in which the maps in the top row and the left square are isomorphisms, completes the identification of the edge homomorphism

$$
E_{0, q}^{2} \rightarrow E_{0,2}^{\infty} \cong F_{0} \underline{\pi}_{\alpha+q}^{G, U^{\prime}} \iota_{*} Z \subset \underline{\pi}_{\alpha+q}^{G, U^{\prime}} \iota_{*} Z
$$

with the map $\widetilde{\sigma}_{\iota}: s_{*}^{\iota} \underline{\pi}_{\alpha+q}^{G, U} Z \rightarrow \underline{\pi}_{\alpha+q}^{G, U^{\prime}} \iota_{*} Z$.

5. An alternative approach to the functor $\iota_{*}$. In this section, we introduce an alternative method for studying the change of universe functor $\iota_{*}$ associated with a linear $G$-isometry $\iota: U \rightarrow U^{\prime}$ between $G$-universes $U$ and $U^{\prime}$. This approach is based on an analysis of the composite functor $\iota^{*} \iota_{*}$. This analysis justifies, and makes computationally useful, the intuition that 
the change of universe functor $\iota_{*}$ is some sort of colimit of suspension functors. In particular, if $Z$ is a $G$-spectrum indexed on $U$ and $\alpha \in R O^{+}(G, U)$, then the results of this section indicate that the equivariant stable homotopy group $\pi_{\alpha}^{G, U^{\prime}} \iota_{*} Z$ of the spectrum $\iota_{*} Z$ can be computed as the colimit of a diagram of homotopy groups $\pi_{\alpha+W}^{G, U} \Sigma^{W} Z$ indexed on an appropriate collection of finite-dimensional $G$-representations $W$ that are contained in $U^{\prime}$ but not in $U$. This colimit description of $\pi_{\alpha}^{G, U^{\prime}} \iota_{*} Z$ can even be extended to a description of the entire spectral sequence of Theorem 1.14(b) as a colimit of a diagram of spectral sequences of the form given by Theorem 1.14(a). These colimit descriptions can be used in several ways. If the representation $W$ is fairly simple, then the homotopy group $\pi_{\alpha+W}^{G, U} \Sigma^{W} Z$ can sometimes be computed fairly easily. This computation can then be used to obtain information about $\pi_{\alpha}^{G, U^{\prime}} \iota_{*} Z$. Example 1.18 and the description given in Proposition 1.16 of the $E^{2}$-term of the spectral sequence of Theorem 1.14(b) were first discovered by doing sample calculations using this approach. On the other hand, the $G$-stable category associated with the universe $U^{\prime}$ may be a significantly nicer category than the one associated with $U$. When this is the case, it may be fairly easy to compute the homotopy group $\pi_{\alpha}^{G, U^{\prime}} \iota_{*} Z$. Information about this group can then be used to draw conclusions about the groups $\pi_{\alpha+W}^{G, U} \Sigma^{W} Z$ for sufficiently large representations $W$. The proof, in the next section, that the "only if" part of Theorem 1.2(a) follows from the "only if" part of Theorem 1.2(b) illustrates this method of deriving information about $\pi_{\alpha+W}^{G, U} \Sigma^{W} Z$ from information about $\pi_{\alpha}^{G, U^{\prime}} \iota_{*} Z$. Perhaps the best way to view the analysis of $\iota_{*}$ offered in this section is that it is a simple-minded, brute-force approach to $\iota_{*}$ that has proven to be a useful complement to the more sophisticated approaches offered in Section 1.

Throughout this section, $U$ is a $G$-universe and $U^{\prime \prime}$ is what might be called a reduced $G$-universe - that is, $U^{\prime \prime}$ is a countably infinite-dimensional $G$-inner product space which contains no trivial representations, but which contains each of its finite-dimensional subrepresentations infinitely often. Let $U^{\prime}=U \oplus U^{\prime \prime}$, and let $\iota: U \rightarrow U^{\prime}$ be the inclusion of $U$ into $U^{\prime}$ as a direct summand. It is easy to find an example of a linear $G$-isometry $\zeta: U \rightarrow U^{\prime \prime \prime}$ between $G$-universes $U$ and $U^{\prime \prime \prime}$ which does not have the simple structure that $\iota$ does, essentially because infinite-dimensional subspaces of infinite-dimensional inner product spaces need not have properly behaved orthogonal complements. Nevertheless, if $U$ and $U^{\prime \prime \prime}$ are two $G$-universes between which there exists a linear $G$-isometry $\zeta: U \rightarrow U^{\prime \prime \prime}$, then there is also a linear $G$-isometry $\xi: U \rightarrow U^{\prime \prime \prime}$ which maps $U$ onto a subuniverse of $U^{\prime \prime \prime}$ which is a direct summand and which has a reduced universe as its orthogonal complement. The functors

$$
\zeta_{*}: \widetilde{h} G \mathcal{S} U \rightarrow \widetilde{h} G \mathcal{S} U^{\prime \prime \prime} \quad \text { and } \quad \xi_{*}: \widetilde{h} G \mathcal{S} U \rightarrow \widetilde{h} G \mathcal{S} U^{\prime \prime \prime}
$$


are naturally isomorphic by Theorem II.1.7 of [18]. Thus, for computational purposes, analyzing the restricted type of isometries $\iota$ discussed here is sufficient.

Recall from Section I.2 of [18] that an indexing sequence for the universe $U$ is an increasing sequence $\left\{A_{m}\right\}_{m \geq 0}$ of finite-dimensional subspaces of $U$ such that $A_{0}=0$ and $U=\bigcup_{m} A_{m}$. Define an indexing sequence for a reduced universe analogously. For the remainder of this section, we select fixed indexing sequences $\left\{A_{m}\right\}_{m \geq 0}$ for $U$ and $\left\{C_{n}\right\}_{n \geq 0}$ for $U^{\prime \prime}$. Note that the collection $\left\{A_{m} \oplus C_{m}\right\}_{m>0}$ forms an indexing sequence for the universe $U^{\prime}$. Moreover, the larger collection $\left\{A_{m} \oplus C_{n}\right\}_{m, n \geq 0}$ forms an indexing set for $U^{\prime}$ in the sense of Section I.2 of [18]. Hereafter, we denote the orthogonal complements of $A_{m}$ in $A_{m+1}$ and $C_{n}$ in $C_{n+1}$ by $B_{m}$ and $D_{n}$, respectively.

If $Z$ is a $G$-spectrum indexed on $U$, then the sequence of spectra $\Omega^{C_{n}} \Sigma^{C_{n}} Z, n \geq 0$, is linked together by the sequence of maps

$$
\Omega^{C_{n}} \Sigma^{C_{n}} Z \stackrel{\Omega^{C_{n}} \eta_{n}}{\longrightarrow} \Omega^{C_{n}} \Omega^{D_{n}} \Sigma^{D_{n}} \Sigma^{C_{n}} Z \cong \Omega^{C_{n+1}} \Sigma^{C_{n+1}} Z,
$$

where $\eta_{n}$ is the unit of the $\left(\Sigma^{D_{n}}, \Omega^{D_{n}}\right)$-adjunction, and the isomorphism is derived from the identification of $C_{n} \oplus D_{n}$ with $C_{n+1}$. Thus, we can form the telescope $\operatorname{Tel}_{n} \Omega^{C_{n}} \Sigma^{C_{n}} Z$, which might be thought of as a homotopytheoretic, spectrum-level generalization of the free infinite loop space construction $\Omega^{\infty} \Sigma^{\infty}$. Our alternative characterization of the functor $\iota_{*}$, or more precisely of $\iota^{*} \iota_{*}$, is in terms of this telescope; it is proved at the end of this section.

THEOREM 5.1. There is an isomorphism

$$
\tau: \iota^{*} \iota_{*} Z \rightarrow \underset{n}{\operatorname{Tel}} \Omega^{C_{n}} \Sigma^{C_{n}} Z
$$

in $\widetilde{h} G \mathcal{S U}$ which is natural in $Z$. Moreover, under this isomorphism, the unit $\eta: Z \rightarrow \iota^{*} \iota_{*} Z$ of the $\left(\iota_{*}, \iota^{*}\right)$-adjunction is identified with the canonical inclusion of $Z=\Omega^{C_{0}} \Sigma^{C_{0}} Z$ into the telescope.

The $\left(\iota_{*}, \iota^{*}\right)$ - and $\left(\Sigma^{C_{n}}, \Omega^{C_{n}}\right)$-adjunctions allow us to use the isomorphism $\tau$ to compute the homotopy groups of the spectrum $\iota_{*} Z$. Let $\alpha$ be in $R O^{+}(G, U)$, and $K$ be a compact $G$-space. Consider the colimit of the sequence of morphism groups $\left[\Sigma^{C_{n}}\left(S_{U}^{\alpha} \wedge K\right), \Sigma^{C_{n}} Z\right]_{G}^{U}$ over the sequence of maps

$$
\begin{aligned}
{\left[\Sigma^{C_{n}}\left(S_{U}^{\alpha} \wedge K\right), \Sigma^{C_{n}} Z\right]_{G}^{U} } & \rightarrow\left[\Sigma^{C_{n}+D_{n}}\left(S_{U}^{\alpha} \wedge K\right), \Sigma^{C_{n}+D_{n}} Z\right]_{G}^{U} \\
& \cong\left[\Sigma^{C_{n+1}}\left(S_{U}^{\alpha} \wedge K\right), \Sigma^{C_{n+1}} Z\right]_{G}^{U}
\end{aligned}
$$

induced by suspension by $D_{n}$. A simple application of Lemma I.4.8 of [18] yields the following computationally useful consequence of our theorem. 
COROLlaRY 5.2. There are isomorphisms

$\left[S_{U^{\prime}}^{\alpha} \wedge K, \iota_{*} Z\right]_{G}^{U^{\prime}} \cong\left[S_{U}^{\alpha} \wedge K, \iota^{*} \iota_{*} Z\right]_{G}^{U} \cong \operatorname{colim}_{n}\left[\Sigma^{C_{n}}\left(S_{U}^{\alpha} \wedge K\right), \Sigma^{C_{n}} Z\right]_{G}^{U}$,

which are natural in $K$ and $Z$. In particular, if $H \leq G$, then there are isomorphisms

$$
\pi_{\alpha}^{H, U^{\prime}} \iota_{*} Z \cong \pi_{\alpha}^{H, U} \iota^{*} \iota_{*} Z \cong \operatorname{colim}_{n} \pi_{\alpha+C_{n}}^{H, U} \Sigma^{C_{n}} Z
$$

which are natural in $Z$. Moreover, under these isomorphisms, the maps

$$
\sigma_{\iota}:\left[S_{U}^{\alpha} \wedge K, Z\right]_{G}^{U} \rightarrow\left[S_{U^{\prime}}^{\alpha} \wedge K, \iota_{*} Z\right]_{G}^{U^{\prime}} \quad \text { and } \quad \sigma_{\iota}: \pi_{\alpha}^{H, U} Z \rightarrow \pi_{\alpha}^{H, U^{\prime}} \iota_{*} Z
$$

induced by $\iota_{*}$ become the inclusions of the first terms of the colimit diagrams into the colimits.

Remark 5.3. If $\alpha$ is in $R O^{+}\left(G, U^{\prime}\right)$ but not in $R O^{+}(G, U)$, then, by a more careful use of adjunctions, Theorem 5.1 can still be used to compute $\pi_{\alpha}^{H, U^{\prime}} \iota_{*} Z$. Represent $\alpha$ by a formal difference $V-W$ in which $W$ imbeds into $U^{\prime}$ up to $G$-orbits. Then

$$
\begin{aligned}
\pi_{\alpha}^{H, U^{\prime}} \iota_{*} Z & =\left[S_{U^{\prime}}^{\alpha} \wedge G / H_{+}, \iota_{*} Z\right]_{G}^{U^{\prime}} \cong\left[S_{U^{\prime}}^{V} \wedge G / H_{+}, \iota_{*} \Sigma^{W} Z\right]_{G}^{U^{\prime}} \\
& \cong \operatorname{colim}_{n} \pi_{V+C_{n}}^{H, U} \Sigma^{C_{n}+W} Z .
\end{aligned}
$$

Thus, all of the $R O^{+}\left(G, U^{\prime}\right)$-graded homotopy groups of $\iota_{*} Z$ may be described in terms of the $R O^{+}(G, U)$-graded homotopy groups of $Z$ and its suspensions.

In order to prove Theorem 5.1, we must delve much more deeply into the construction of the category $\widetilde{h} G \mathcal{S} U$ than we have before. Recall from Chapter I of [18] that the stable category $\widetilde{h} G \mathcal{S} U$ associated with a $G$-universe $U$ is obtained from a category $G \mathcal{S} U$ of $G$-spectra and actual $G$-maps of $G$-spectra by passing to the homotopy category $h G \mathcal{S} U$ of $G \mathcal{S} U$ and then inverting weak equivalences. The category $G \mathcal{S} U$ is a subcategory of the category $G \mathcal{P} U$ of $G$-prespectra indexed on $U$, and there is a left adjoint $L: G \mathcal{P} U \rightarrow G \mathcal{S} U$, called the spectrafication functor, to the inclusion of $G \mathcal{S} U$ into $G \mathcal{P} U$. The spectrum-level change of universe functor $\iota_{*}: G \mathcal{S} U \rightarrow G \mathcal{S} U^{\prime}$ and suspension functor $\Sigma^{W}: G \mathcal{S} U \rightarrow G \mathcal{S} U$ are obtained by applying the spectrafication functors $L^{\prime}: G \mathcal{P} U^{\prime} \rightarrow G \mathcal{S} U^{\prime}$ and $L: G \mathcal{P} U \rightarrow G \mathcal{S} U$ to a prespectrum-level change of universe functor $\iota_{\mathcal{P}}: G \mathcal{P} U \rightarrow G \mathcal{P} U^{\prime}$ and suspension functor $\Sigma_{\mathcal{P}}^{W}: G \mathcal{P} U \rightarrow G \mathcal{P} U$. In order to define $\iota_{\mathcal{P}}$ and $\Sigma_{\mathcal{P}}^{W}$, we assume hereafter that all prespectra associated with the universe $U$ are indexed on our standard indexing sequence $\left\{A_{m}\right\}$ and that all prespectra associated with $U^{\prime}$ are indexed on the indexing set $\left\{A_{m}+C_{n}\right\}$. If $X$ is a prespectrum indexed on $U$, then the component spaces of the prespectra $\iota_{\mathcal{P}} X$ and $\Sigma_{\mathcal{P}}^{W} X$ are given by

$$
\left(\iota_{\mathcal{P}} X\right)\left(A_{m}+C_{n}\right)=\Sigma^{C_{n}} X A_{m} \quad \text { and } \quad\left(\Sigma_{\mathcal{P}}^{W} X\right)\left(A_{m}\right)=\Sigma^{W} X A_{m} .
$$


The structure maps of $\iota_{\mathcal{P}} X$ and $\Sigma_{\mathcal{P}}^{W} X$ are defined in the obvious way (see Definitions II.1.1 and I.3.1 of [18]). A prespectrum $X$ indexed on the universe $U$ is said to be a $\Sigma$-inclusion prespectrum if, for every integer $m$, the structure map $\varsigma: \Sigma^{B_{m}} X A_{m} \rightarrow X A_{m+1}$ of $X$ is a closed inclusion (see Definitions I.8.2 of [18]). For our present purposes, $\Sigma$-inclusion prespectra have several advantages over arbitrary prespectra. The first advantage is that, if $X$ is a $\Sigma$-inclusion prespectrum indexed on $U$, then the spectrum $L X$ is given by

$$
(L X)\left(A_{m}\right)=\operatorname{colim}_{p \geq m} \Omega^{A_{p}-A_{m}} X A_{p},
$$

where the colimit is taken over the obvious sequence of maps derived from the structure maps of $X$. Here, and hereafter in this section, $A_{p}-A_{m}$ denotes the orthogonal complement of $A_{m}$ in $A_{p}$. It follows easily from this description of $L$ that, if $f: X \rightarrow Y$ is a map between two $\Sigma$-inclusion prespectra which is a spacewise weak equivalence (that is, $f A_{m}: X A_{m} \rightarrow Y A_{m}$ is a weak equivalence of based $G$-spaces for each integer $m$ ), then the map $L f: L X \rightarrow L Y$ is a weak equivalence of spectra. If the prespectrum $X$ is not a $\Sigma$-inclusion prespectrum, then $L X$ does not have such a simple description. Moreover, $L$ need not convert a spacewise weak equivalence between arbitrary prespectra into a weak equivalence between spectra. A second advantage of $\Sigma$-inclusion prespectra is that the prespectrum-level functors $\iota_{\mathcal{P}}$ and $\Sigma_{\mathcal{P}}^{W}$ take $\Sigma$-inclusion prespectra to $\Sigma$-inclusion prespectra. Thus, if $X$ is a $\Sigma$-inclusion prespectrum, then the spectra $\iota_{*} L X \cong L \iota_{\mathcal{P}} X$ and $\Sigma^{W} L X \cong L \Sigma_{\mathcal{P}}^{W} X$ have relatively simple descriptions. These descriptions allow us to prove the following result, from which we derive Theorem 5.1.

Proposition 5.4. Let $X$ be a $\Sigma$-inclusion $G$-prespectrum indexed on the indexing sequence $\left\{A_{m}\right\}$ for the universe $U$. Then there is an isomorphism

$$
\iota^{*} \iota_{*} L X \cong \operatorname{colim}_{n} \Omega^{C_{n}} \Sigma^{C_{n}} L X
$$

in GSU, which is natural in X. Moreover, under this isomorphism, the unit $\eta: L X \rightarrow \iota^{*} \iota_{*} L X$ of the $\left(\iota_{*}, \iota^{*}\right)$-adjunction is identified with the canonical inclusion of the spectrum $L X=\Omega^{C_{0}} \Sigma^{C_{0}} L X$ into the colimit.

Proof. Since $\Sigma^{C_{n}} L X \cong L \Sigma_{\mathcal{P}}^{C_{n}} X$ and $\iota_{*} L X \cong L \iota_{\mathcal{P}} X$, the asserted isomorphism may be derived from an isomorphism between $\iota^{*} L \iota_{\mathcal{P}} X$ and $\operatorname{colim}_{n} \Omega^{C_{n}} L \Sigma_{\mathcal{P}}^{C_{n}} X$. The colimit here is intended to be taken in the category $G \mathcal{S U}$ of spectra. Colimits in the category of spectra are generally obtained by applying $L$ to the corresponding colimits in the category of prespectra. Since $L$ can be rather badly behaved, colimits in the category of spectra can also be rather badly behaved. However, here we show that the prespectrum-level colimit of the spectra $\Omega^{C_{n}} L \Sigma_{\mathcal{P}}^{C_{n}} X$ is isomorphic to the spectrum $\iota^{*} L \iota_{\mathcal{P}} X$. This prespectrum-level colimit, being a spectrum, is 
therefore also the spectrum-level colimit, and we need not apply $L$. From the descriptions of $L$ and $\iota_{\mathcal{P}}$ given above, we obtain an isomorphism

$$
\left(\iota^{*} L \iota_{\mathcal{P}} X\right)\left(A_{m}\right)=\left(L \iota_{\mathcal{P}} X\right)\left(A_{m}+0\right) \cong \operatorname{colim}_{n, p \geq m} \Omega^{\left(A_{p}-A_{m}\right)+C_{n}} \Sigma^{C_{n}} X A_{p},
$$

for each $m \geq 0$. However,

$$
\begin{aligned}
\operatorname{colim}_{n, p \geq m} \Omega^{\left(A_{p}-A_{m}\right)+C_{n}} \Sigma^{C_{n}} X A_{p} & \cong \operatorname{colim}_{n} \operatorname{colim}_{p \geq m} \Omega^{C_{n}} \Omega^{\left(A_{p}-A_{m}\right)} \Sigma^{C_{n}} X A_{p} \\
& \cong \operatorname{colim}_{n} \Omega^{C_{n}}\left(\operatorname{colim}_{p \geq m} \Omega^{\left(A_{p}-A_{m}\right)} \Sigma^{C_{n}} X A_{p}\right) .
\end{aligned}
$$

Here, the functor $\Omega^{C_{n}}$ commutes with the colimits because the space $S^{C_{n}}$ is compact and the colimits are taken over sequences of maps which are inclusions. The space $\operatorname{colim}_{p \geq m} \Omega^{\left(A_{p}-A_{m}\right)} \Sigma^{C_{n}} X A_{p}$ is just the component space $\left(L \Sigma_{\mathcal{P}}^{C_{n}} X\right)\left(A_{m}\right)$ of the spectrum $L \Sigma_{\mathcal{P}}^{C_{n}} X$. Thus, the $m$ th component space of the prespectrum-level colimit $\operatorname{colim}_{n} \Omega^{C_{n}} L \Sigma_{\mathcal{P}}^{C_{n}} X$ is also described by an isomorphism

$$
\left(\operatorname{colim}_{n} \Omega^{C_{n}} L \Sigma_{\mathcal{P}}^{C_{n}} X\right)\left(A_{m}\right) \cong \operatorname{colim}_{n} \Omega^{C_{n}}\left(\operatorname{colim}_{p \geq m} \Omega^{\left(A_{p}-A_{m}\right)} \Sigma^{C_{n}} X A_{p}\right) .
$$

Combining these identifications, we obtain an isomorphism

$$
\gamma_{m}:\left(\iota^{*} L \iota_{\mathcal{P}} X\right)\left(A_{m}\right) \cong\left(\operatorname{colim}_{n} \Omega^{C_{n}} L \Sigma_{\mathcal{P}}^{C_{n}} X\right)\left(A_{m}\right)
$$

between the component spaces of the spectrum $\iota^{*} L \iota_{\mathcal{P}} X$ and the prespectrum $\operatorname{colim}_{n} \Omega^{C_{n}} L \Sigma_{\mathcal{P}}^{C_{n}} X$. It is easy to check that the isomorphisms $\gamma_{m}$, for $m \geq 0$, commute with the structure maps of these two prespectra. It follows that the prespectrum-level colimit $\operatorname{colim}_{n} \Omega^{C_{n}} L \Sigma_{\mathcal{P}}^{C_{n}} X$ is a spectrum and is isomorphic, in $G \mathcal{S} U$, to the spectrum $\iota^{*} L \iota_{\mathcal{P}} X$. It is relatively easy to chase through the sequence of isomorphisms given above and verify the asserted relationship between the unit $\eta: L X \rightarrow \iota^{*} \iota_{*} L X$ of the $\left(\iota_{*}, \iota^{*}\right)$-adjunction and the canonical inclusion of the spectrum $L X=\Omega^{C_{0}} \Sigma^{C_{0}} L X$ into the colimit.

Proof of Theorem 5.1. Let $Z$ be a $G$-spectrum indexed on $U$. By Propositions I.8.13 and I.8.14 of [18], there is a $\Sigma$-inclusion $G$-prespectrum $X$ and a map $f: X \rightarrow Z$ of $G$-prespectra such that the spectrum $L X$ has the $G$-homotopy type of a $G-C W$ spectrum and the map $f: L X \rightarrow Z$ induced by $f$ is a weak equivalence. The natural projection

$$
\underset{n}{\mathrm{Tel}} \Omega^{C_{n}} \Sigma^{C_{n}} L X \rightarrow \operatorname{colim}_{n} \Omega^{C_{n}} \Sigma^{C_{n}} L X
$$

is a weak equivalence in $G \mathcal{S} U$ because, as indicated in the proof of the proposition above, the colimit $\operatorname{colim}_{n} \Omega^{C_{n}} \Sigma^{C_{n}} L X$ may be formed as a prespectrum-level colimit over a sequence of maps that are spacewise inclusions. Moreover, the map

$$
\underset{n}{\mathrm{Tel}} \Omega^{C_{n}} \Sigma^{C_{n}} L X \rightarrow \mathrm{Tel}_{n} \Omega^{C_{n}} \Sigma^{C_{n}} Z,
$$


induced by the map $\widetilde{f}$, is a weak equivalence since $\widetilde{f}$ is. These two weak equivalences, combined with the composite

$$
\underset{n}{\operatorname{colim}} \Omega^{C_{n}} \Sigma^{C_{n}} L X \cong \iota^{*} \iota_{*} L X \stackrel{\iota^{*} \iota_{*} \tilde{f}}{\longrightarrow} \iota^{*} \iota_{*} Z,
$$

yield the isomorphism in $\widetilde{h} G \mathcal{S} U$ of the theorem. The assertion in the theorem about the relation between the unit $\eta: Z \rightarrow \iota^{*} \iota_{*} Z$ of the $\left(\iota_{*}, \iota^{*}\right)$ adjunction and the canonical inclusion of $Z=\Omega^{C_{0}} \Sigma^{C_{0}} Z$ into the telescope $\mathrm{Tel}_{n} \Omega^{C_{n}} \Sigma^{C_{n}} Z$ follows from the analogous assertion in Proposition 5.4.

6. The proof of Theorem 1.2. We have already noted that the "if" portion of Theorem 1.2(a) follows from Theorem 1.2(b). In this section, we give a counterexample to establish the "only if" portions of both part (a) and part (b) of the theorem and then prove the "if" portion of part (b).

The following example proves the "only if" portion of Theorem 1.2(b) by showing that, if $\iota: U \rightarrow U^{\prime}$ is a linear $G$-isometry between two $G$-universes that are not $G$-orbit equivalent, then the map

$$
\sigma_{\iota}:\left[S_{U}^{n} \wedge G / H_{+}, S_{U}^{0}\right]_{G}^{U} \rightarrow\left[\iota_{*} S_{U}^{n} \wedge G / H_{+}, \iota_{*} S_{U}^{0}\right]_{G}^{U^{\prime}} \cong\left[S_{U^{\prime}}^{n} \wedge G / H_{+}, S_{U^{\prime}}^{0}\right]_{G}^{U^{\prime}}
$$

is not onto for some subgroup $H$ of $G$ and some positive integer $n$.

ExAmple 6.1. Let $\iota: U \rightarrow U^{\prime}$ be a linear $G$-isometry between two $G$-universes that are not $G$-orbit equivalent. Since $\iota$ is an equivariant isometry, $U$ must be contained in $U^{\prime}$ up to $G$-orbit equivalence. Thus, $U^{\prime}$ cannot be contained in $U$ up to $G$-orbit equivalence, and there exist a pair of subgroups $K \leq H$ of $G$ such that $H / K H$-imbeds in $U^{\prime}$ but does not $H$-imbed in $U$. Hereafter, we refer to such a subgroup $K$ as a bad subgroup of $H$. We wish to show that, for this subgroup $H$, there is a positive integer $n$ such that the map

$$
\sigma_{\iota}:\left[S_{U}^{n} \wedge G / H_{+}, S_{U}^{0}\right]_{G}^{U} \rightarrow\left[S_{U^{\prime}}^{n} \wedge G / H_{+}, S_{U^{\prime}}^{0}\right]_{G}^{U^{\prime}}
$$

is not onto. Under the change of group isomorphisms (see Theorem II.4.7 and Lemma II.4.8 of [18])

$\left[S_{U}^{n} \wedge G / H_{+}, S_{U}^{0}\right]_{G}^{U} \cong\left[S_{U}^{n}, S_{U}^{0}\right]_{H}^{U} \quad$ and $\quad\left[S_{U^{\prime}}^{n} \wedge G / H_{+}, S_{U^{\prime}}^{0}\right]_{G}^{U^{\prime}} \cong\left[S_{U^{\prime}}^{n}, S_{U^{\prime}}^{0}\right]_{H}^{U^{\prime}}$,

this map is identified with the map

$$
\sigma_{\iota}:\left[S_{U}^{n}, S_{U}^{0}\right]_{H}^{U} \rightarrow\left[S_{U^{\prime}}^{n}, S_{U^{\prime}}^{0}\right]_{H}^{U^{\prime}}
$$

induced by the change of universe functor $\iota_{*}: \widetilde{h} H \mathcal{S} U \rightarrow \widetilde{h} H \mathcal{S} U^{\prime}$. The group $\left[S_{U}^{0}, S_{U}^{0}\right]_{H}^{U}$ is just the free abelian group generated by the equivariant Euler characteristics of the orbits $H / J$ such that $H / J H$-imbeds in $U$ and $J$ has finite index in its $H$-normalizer $N J$. The map

$$
\sigma_{\iota}:\left[S_{U}^{0}, S_{U}^{0}\right]_{H}^{U} \rightarrow\left[S_{U^{\prime}}^{0}, S_{U^{\prime}}^{0}\right]_{H}^{U^{\prime}}
$$


simply takes these generators in $\left[S_{U}^{0}, S_{U}^{0}\right]_{H}^{U}$ to the corresponding generators in $\left[S_{U^{\prime}}^{0}, S_{U^{\prime}}^{0}\right]_{H}^{U^{\prime}}$. Thus, if there is a bad subgroup $K$ of $H$ that has finite index in its $H$-normalizer $N K$, then the equivariant Euler characteristic of $H / K$ is a nonzero element of $\left[S_{U^{\prime}}^{0}, S_{U^{\prime}}^{0}\right]_{H}^{U^{\prime}}$ that is not in the image of $\sigma_{\iota}$. If no bad subgroup $K$ of $H$ has finite index in its $H$-normalizer, then we must make use of the splitting introduced in [15] to produce an example in which $\sigma_{\iota}$ is not an isomorphism. By that splitting, for any positive integer $n$,

$$
\left[S_{U}^{n}, S_{U}^{0}\right]_{H}^{U} \cong \bigoplus \pi_{n}\left(\Sigma^{\infty} E W J_{+} \wedge_{W J} S^{A(J)}\right),
$$

where the sum runs over the $H$-conjugacy classes of subgroups $J$ of $H$ such that $H / J H$-imbeds in $U$. Here, $W J$ is the Weyl group $N J / J$ of $J, A(J)$ is the adjoint representation of $W J$, and the homotopy groups on the right are just nonequivariant stable homotopy groups. For each subgroup $J$, the space $E W J_{+} \wedge_{W J} S^{A(J)}$ is the Thom space of a bundle over the classifying space $B W J$. It has a lowest-dimensional nonvanishing stable homotopy group that must be either $\mathbb{Z}$ or $\mathbb{Z} / 2$ (see Lemma IX.1.6 of [18]). The equivariant stable homotopy group $\left[S_{U^{\prime}}^{n}, S_{U^{\prime}}^{0}\right]_{H}^{U^{\prime}}$ splits in a similar fashion. With respect to these splittings, the map $\sigma_{\iota}$ is just the inclusion of those summands of $\left[S_{U^{\prime}}^{n}, S_{U^{\prime}}^{0}\right]_{H}^{U^{\prime}}$ indexed on the $H$-conjugacy classes of subgroups $J$ of $H$ such that $H / J$ $H$-imbeds in both $U$ and $U^{\prime}$. Clearly, the summand of $\left[S_{U^{\prime}}^{n}, S_{U^{\prime}}^{0}\right]_{H}^{U^{\prime}}$ indexed on the conjugacy class of the bad subgroup $K$ of $H$ is not in the image of $\sigma_{\iota}$. This summand is either $\mathbb{Z}$ or $\mathbb{Z} / 2$ for some integer $n$, and $\sigma_{\iota}$ is therefore not onto.

The "only if" part of Theorem 1.2(a) follows from this example and Corollary 5.2. Assume that $W$ is a finite-dimensional $G$-representation that is not contained in $U$ up to $G$-orbit type. Let $U^{\prime}$ be the direct sum of $U$ and countably infinitely many copies of $W$, and let $\iota: U \rightarrow U^{\prime}$ be the inclusion of $U$ into $U^{\prime}$ as a direct summand. Then $U$ and $U^{\prime}$ cannot be $G$-orbit equivalent. Therefore, by the example above, the map

$$
\sigma_{\iota}:\left[S_{U}^{n} \wedge G / H_{+}, S_{U}^{0}\right]_{G}^{U} \rightarrow\left[\iota_{*} S_{U}^{n} \wedge G / H_{+}, \iota_{*} S_{U}^{0}\right]_{G}^{U^{\prime}} \cong\left[S_{U^{\prime}}^{n} \wedge G / H_{+}, S_{U^{\prime}}^{0}\right]_{G}^{U^{\prime}}
$$

is not onto for some subgroup $H$ of $G$ and some positive integer $n$. However, Corollary 5.2 describes the group $\left[S_{U^{\prime}}^{n} \wedge G / H_{+}, S_{U^{\prime}}^{0}\right]_{G}^{U^{\prime}}$ as the colimit of the groups $\left[\Sigma^{m W}\left(S_{U}^{n} \wedge G / H_{+}\right), S_{U}^{m W}\right]_{G}^{U}$, for $m \geq 0$, over the maps

$$
\left[\Sigma^{m W}\left(S_{U}^{n} \wedge G / H_{+}\right), S_{U}^{m W}\right]_{G}^{U} \rightarrow\left[\Sigma^{(m+1) W}\left(S_{U}^{n} \wedge G / H_{+}\right), S_{U}^{(m+1) W}\right]_{G}^{U}
$$

induced by the suspension functor $\Sigma^{W}$. In terms of this description, the map $\sigma_{\iota}$ is just the inclusion of the group $\left[S_{U}^{n} \wedge G / H_{+}, S_{U}^{0}\right]_{G}^{U}$ into the colimit. If the functor $\Sigma^{W}$ were invertible, then this colimit would be the colimit of a diagram of isomorphisms, and the inclusion of the group $\left[S_{U}^{n} \wedge G / H_{+}, S_{U}^{0}\right]_{G}^{U}$ into the colimit would be an isomorphism, contradicting the fact that $\sigma_{\iota}$ is not onto. 
The following proposition is the key to proving the "if" part of Theorem $1.2(\mathrm{~b})$.

Proposition 6.2. Let $\iota: U \rightarrow U^{\prime}$ be a linear $G$-isometry between $G$-universes $U$ and $U^{\prime}$ which are $G$-orbit equivalent. Then

(a) For every pair of $G$-spectra $X, Y$ indexed on $U$, the map

$$
\sigma_{\iota}:[X, Y]_{G}^{U} \rightarrow\left[\iota_{*} X, \iota_{*} Y\right]_{G}^{U^{\prime}},
$$

induced by the change of universe functor $\iota_{*}$, is an isomorphism.

(b) For every $G$-spectrum $Z$ indexed on $U^{\prime}$, there is a $G$-spectrum $Y$ indexed on $U$ such that $\iota_{*} Y$ and $Z$ are isomorphic in $\widetilde{h} G \mathcal{S} U^{\prime}$.

Proof. For part (a), the commuting diagram

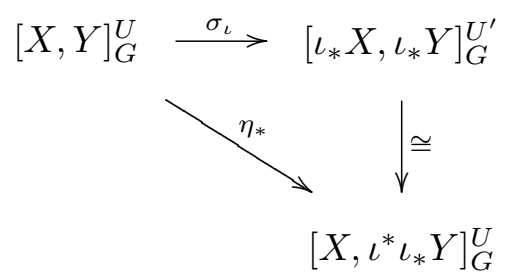

indicates that it suffices to prove that the unit $\eta$ of the $\left(\iota_{*}, \iota^{*}\right)$-adjunction is an isomorphism in $\widetilde{h} G \mathcal{S} U$. To verify this, it suffices to show that either of the maps $\sigma_{\iota}$ or $\eta_{*}$ in the diagram is an isomorphism whenever $X$ is an equivariant sphere spectrum $S_{U}^{q} \wedge G / H_{+}$, where $q \in \mathbb{Z}$ and $H \leq G$. A change of groups isomorphism (see Theorem II.4.7 and Lemma II.4.8 of [18]) reduces this to showing that the map

$$
\sigma_{\iota}:\left[S_{U}^{q}, Y\right]_{H}^{U} \rightarrow\left[\iota_{*} S_{U}^{q}, \iota_{*} Y\right]_{H}^{U^{\prime}} \cong\left[S_{U^{\prime}}^{q}, \iota_{*} Y\right]_{H}^{U^{\prime}}
$$

is an isomorphism. We can now replace $Y$ by an isomorphic $H-C W$ spectrum and argue by induction over its skeleton. This reduces the problem to showing that, for each pair of subgroups $K \leq H$ of $G$ and each integer $q$, the map

$$
\sigma_{\iota}:\left[S_{U}^{q}, \Sigma_{U}^{\infty} H / K_{+}\right]_{H}^{U} \rightarrow\left[\iota_{*} S_{U}^{q}, \iota_{*} \Sigma_{U}^{\infty} H / K_{+}\right]_{H}^{U^{\prime}} \cong\left[S_{U^{\prime}}^{q}, \Sigma_{U^{\prime}}^{\infty} H / K_{+}\right]_{H}^{U^{\prime}}
$$

is an isomorphism. Recall now from the remarks at the beginning of Section 5 that we can assume that $U^{\prime}$ is the direct sum of $U$ and a reduced $G$-universe $U^{\prime \prime}$, and that $\iota$ is the inclusion of $U$ into $U^{\prime}$ as a direct summand. As in Section 5, we select indexing sequences $\left\{A_{m}\right\}_{m \geq 0}$ for $U$ and $\left\{C_{n}\right\}_{n \geq 0}$ for $U^{\prime \prime}$, and adopt the collection $\left\{A_{m} \oplus C_{n}\right\}_{m, n \geq 0}$ as an indexing set for $U^{\prime}$. Then, by Corollary I.4.9 of [18], we have isomorphisms

$$
\left[S_{U}^{q}, \Sigma_{U}^{\infty} H / K_{+}\right]_{H}^{U} \cong \operatorname{colim}_{n}\left[S^{q+A_{m}}, \Sigma^{A_{m}} H / K_{+}\right]_{H}
$$


and

$$
\left[S_{U^{\prime}}^{q}, \Sigma_{U^{\prime}}^{\infty} H / K_{+}\right]_{H}^{U^{\prime}} \cong \operatorname{colim}_{m, n}\left[S^{q+A_{m}+C_{n}}, \Sigma^{A_{m}+C_{n}} H / K_{+}\right]_{H},
$$

where, in each case, the morphism sets on the right hand side are in the homotopy category of based $H$-spaces. Under these isomorphisms, the map $\sigma_{\iota}:\left[S_{U}^{q}, \Sigma_{U}^{\infty} H / K_{+}\right]_{H}^{U} \rightarrow\left[S_{U^{\prime}}^{q}, \Sigma_{U^{\prime}}^{\infty} H / K_{+}\right]_{H}^{U^{\prime}}$ is obtained by passage to colimits from the maps

$$
\sigma_{m, n}:\left[S^{q+A_{m}}, \Sigma^{A_{m}} H / K_{+}\right]_{H} \rightarrow\left[S^{q+A_{m}+C_{n}}, \Sigma^{A_{m}+C_{n}} H / K_{+}\right]_{H}
$$

induced by the space-level suspension functors $\Sigma^{C_{n}}$. Since the universes $U$ and $U^{\prime}$ are $G$-orbit equivalent, the representations $C_{n}$ must be contained in $U$ up to $G$-orbits. The classical equivariant suspension theorem $[4,7,24,27]$ therefore indicates that, for each nonnegative integer $n$, there is a positive integer $M_{n}$ such that, if $m \geq M_{n}$, then the map $\sigma_{m, n}$ is an isomorphism. Thus, on a cofinal portion of the relevant colimit diagrams, the maps $\sigma_{m, n}$ are isomorphisms. It follows that the induced map $\sigma_{\iota}$ between the colimits is an isomorphism.

To prove part (b), we can assume that $Z$ is a $G-C W$ spectrum indexed on $U^{\prime}$ (see Section I.5 of [18]). Since the functor $\iota_{*}$ takes the equivariant sphere spectra $S_{U}^{q} \wedge G / H_{+}$indexed on $U$ to the corresponding equivariant sphere spectra $S_{U^{\prime}}^{q} \wedge G / H_{+}$indexed on $U^{\prime}$ and the map $\sigma_{\iota}$ of part (a) is an isomorphism, it is possible to work inductively up the skeleton of $Z$ to construct a $G-C W$ spectrum $Y$ indexed on $U$ such that $\iota_{*} Y$ is isomorphic to $Z$ in $\widetilde{h} G \mathcal{S} U^{\prime}$.

A few categorical formalities now complete the proof of the "if" portion of Theorem 1.2(b).

Proof (of the sufficiency of $G$-orbit equivalence in Theorem 1.2(b)). Assume that $\iota: U \rightarrow U^{\prime}$ is a linear $G$-isometry between two $G$-universes $U$ and $U^{\prime}$ which are $G$-orbit equivalent. To show that the functors

$$
\iota_{*}: \widetilde{h} G \mathcal{S} U \rightarrow \widetilde{h} G \mathcal{S} U^{\prime} \quad \text { and } \quad \iota^{*}: \widetilde{h} G \mathcal{S} U^{\prime} \rightarrow \widetilde{h} G \mathcal{S} U
$$

are inverse equivalences of categories, it suffices to show that the unit $\eta: Y \rightarrow \iota^{*} \iota_{*} Y$ and counit $\varepsilon: \iota_{*} \iota^{*} Z \rightarrow Z$ of the $\left(\iota_{*}, \iota^{*}\right)$-adjunction are natural isomorphisms. In the proof of Proposition 6.2, we showed that $\eta$ is a natural isomorphism. The triangle identity

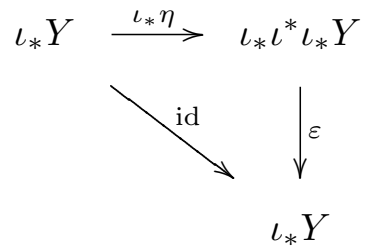


for the adjunction indicates that the counit $\varepsilon: \iota_{*} \iota^{*} \iota_{*} Y \rightarrow \iota_{*} Y$ is an isomorphism for any $G$-spectrum $Y$ indexed on $U$. Part (b) of the proposition indicates that each $G$-spectrum $Z$ indexed on $U^{\prime}$ is isomorphic to a spectrum of the form $\iota_{*} Y$ for some $G$-spectrum $Y$ indexed on $U$. Thus, $\varepsilon$ is always an isomorphism.

\section{References}

[1] G. E. Bredon, Equivariant Cohomology Theories, Lecture Notes in Math. 34, Springer, 1967.

[2] D. Burghelea and A. Deleanu, The homotopy category of spectra. II, Math. Ann. 178 (1968), 131-144.

[3] S. R. Costenoble, S. Waner and G. S. Wells, Approximating equivariant mapping spaces, Pacific J. Math. 144 (1990), 15-45.

[4] T. tom Dieck, Transformation Groups, Walter de Gruyter, Berlin, 1987.

[5] A. Dress, Contributions to the theory of induced representations, in: Lecture Notes in Math. 342, Springer, 1973, 183-240.

[6] J. P. C. Greenlees and J. P. May, Generalized Tate cohomology, Mem. Amer. Math. Soc. 543 (1995).

[7] H. Hauschild, Äquivariante Homotopie I, Arch. Math. (Basel) 29 (1977), 158-165.

[8] —, Äquivariante Konfigurationsräume und Abbildungsräume, in: Lecture Notes in Math. 788, Springer, 1980, 281-315.

[9] —, Zerspaltung äquivarianter Homotopiemengen, Math. Ann. 230 (1977), 279-292.

[10] S. Illman, Equivariant singular homology and cohomology I, Mem. Amer. Math. Soc. 156 (1975).

[11] L. G. Lewis, Jr., An introduction to Mackey functors, in preparation.

[12] -, Equivariant Eilenberg-MacLane spaces and the equivariant Seifert-van Kampen and suspension theorems, Topology Appl. 48 (1992), 25-61.

[13] —, The equivariant Hurewicz map, Trans. Amer. Math. Soc. 329 (1992), 433-472.

[14] - , The $R O(G)$-graded equivariant ordinary cohomology of complex projective spaces with linear $\mathbb{Z} / p$ actions, in: Lecture Notes in Math. 1361, Springer, 1988, 53-122.

[15] —, Splitting theorems for certain equivariant spectra, submitted.

[16] - The stable category and generalized Thom spectra, thesis, Univ. of Chicago, 1978.

[17] L. G. Lewis, Jr., J. P. May and J. E. McClure, Ordinary RO(G)-graded cohomology, Bull. Amer. Math. Soc. 4 (1981), 208-212.

[18] L. G. Lewis, Jr., J. P. May and M. Steinberger (with contributions by J. E. McClure), Equivariant Stable Homotopy Theory, Lecture Notes in Math. 1213, Springer, 1986.

[19] H. R. Margolis, Spectra and the Steenrod Algebra, North-Holland, New York, 1983.

[20] J. P. May, The dual Whitehead theorems, in: London Math. Soc. Lecture Note Ser. 86, Cambridge Univ. Press, 1983, 46-54.

[21] - The homotopical foundations of algebraic topology, in preparation.

[22] J. McCleary, User's Guide to Spectral Sequences, Publish or Perish, 1985.

[23] M. C. McCord, Classifying spaces and infinite symmetric products, Trans. Amer. Math. Soc. 146 (1969), 273-298.

[24] U. Namboodiri, Equivariant vector fields on spheres, ibid. 278 (1983), 431-460.

[25] J. Thévenaz, A visit to the kingdom of the Mackey functors, in: Darstellungstheorietage (Sion, 1989), Bayreuth. Math. Schr. 33 (1990), 215-241. 
[26] A. Turull, Characterizing modules by the dimensions of fixed points, J. Algebra 79 (1982), 248-249.

[27] S. Waner, $G-C W(V)$ complexes, unpublished manuscript.

[28] -, Mackey functors and G-cohomology, Proc. Amer. Math. Soc. 90 (1984), 641-648.

[29] O. Wyler, Convenient categories for topology, Gen. Topology Appl. 3 (1973), 225242 .

DEPARTMENT OF MATHEMATICS

SYRACUSE UNIVERSITY

SYRACUSE, NEW YORK 13244-1150

U.S.A.

E-mail: GAUNCE@ICHTHUS.SYR.EDU

Received 6 January 1995;

in revised form 21 June 1995 